\title{
Heme-deficient metabolism and impaired cellular differentiation as an evolutionary trade-off for human infectivity in Trypanosoma brucei gambiense
}

Eva Horáková ( $\square$ horakova@paru.cas.cz )

Biology Centre

Laurence Lecordier

Université Libre de Bruxelles

Paula Cunha

Laboratory of Neurovascular Signaling, Department of Molecular Biology, Université libre de Bruxelles (ULB)

\section{Roman Sobotka}

Institute of Microbiology https://orcid.org/0000-0001-5909-3879

Piya Changmai

Biology Centre

Catharina Langedijk

Biology Centre

Jan Van Den Abbeele

Institute of Tropical Medicine

\section{Benoit Vanhollebeke}

Université https://orcid.org/0000-0002-0353-365X

Julius Lukes

Czech Academy of Sciences, Institute of Parasitology https://orcid.org/0000-0002-0578-6618

\section{Article}

Keywords: African trypanosomes, trypanosome haptoglobin-hemoglobin receptor, Trypanosoma brucei gambiense

Posted Date: September 8th, 2020

DOI: https://doi.org/10.21203/rs.3.rs-49796/v1

License: (1) This work is licensed under a Creative Commons Attribution 4.0 International License. Read Full License 
Additional Declarations: There is NO Competing Interest.

Version of Record: A version of this preprint was published at Nature Communications on November 18th, 2022. See the published version at https://doi.org/10.1038/s41467-022-34501-4. 
1 Heme-deficient metabolism and impaired cellular differentiation as an evolutionary trade-

3

4

Eva Horáková1,\#,", Laurence Lecordier ${ }^{2, \#}$, Paula Cunha ${ }^{2, \#, \%, ~ R o m a n ~ S o b o t k a ~}{ }^{3,4}$, Piya

Changmai $^{1, \S}$, Catharina J. M. Langedijk ${ }^{1, \&}$, Jan Van Den Abbeele ${ }^{5}$, Benoit Vanhollebeke $^{2,6}$ \& Julius Lukeš ${ }^{1,3, *}$

${ }^{1}$ Institute of Parasitology, Biology Centre, Czech Academy of Sciences, České Budějovice (Budweis), Czech Republic

${ }^{2}$ Laboratory of Neurovascular Signaling, Department of Molecular Biology, Université libre de Bruxelles (ULB), Gosselies, Belgium

${ }^{3}$ Faculty of Sciences, University of South Bohemia, České Budějovice (Budweis), Czech Republic

${ }^{4}$ Institute of Microbiology, Czech Academy of Sciences, Třeboň, Czech Republic

${ }^{5}$ Unit of Veterinary Protozoology, Institute of Tropical Medicine, Antwerp, Belgium

${ }^{6}$ Walloon Excellence in Life Sciences and Biotechnology, Belgium

$\S$ Present address: Faculty of Science, University of Ostrava, Ostrava, Czech Republic

\& Present address: Cancer Center Amsterdam UMC, VU. University Medical Center, Amsterdam, The Netherlands

\% Present address: Escola Paulista de Medicina, Universidade Federal de São Paulo, São Paulo, Brazil

\# These authors contributed equally to the work

*To whom correspondence should be addressed:

Eva Horáková - horakova@paru.cas.cz; Julius Lukeš - jula@paru.cas.cz 


\section{ABSTRACT}

Resistance to African trypanosomes in humans relies on high affinity targeting of a trypanosome lytic factor 1 (TLF1) to trypanosome haptoglobin-hemoglobin receptor ( $\mathrm{HpHbR})$. While TLF1 avoidance by the inactivation of the $\mathrm{HpHbR}$ contributes to Trypanosoma brucei gambiense human infectivity, the evolutionary trade-off of this adaptation is unknown, as the physiological function of the receptor remains to be elucidated. Here we show that uptake of hemoglobin via $\mathrm{HpHbR}$ constitutes the sole heme import pathway in the bloodstream stage of the animal trypanosome T. brucei brucei. Both T. b. gambiense with inactive $\mathrm{HpHbR}$, as well as a genetically engineered $T$. b. brucei $\mathrm{HpHbR}$ knock-out show only trace levels of intracellular heme and lack the downstream hemoprotein activities, thereby providing an extraordinary example of aerobic parasite proliferation in the absence of heme. We further show that $\mathrm{HpHbR}$ facilitates the developmental progression by inducing PAD-1 expression that is associated with the formation of cell cycle-arrested stumpy forms in T. b. brucei. Accordingly, T. b. gambiense was found to be poorly competent for slender-to-stumpy differentiation unless a functional $\mathrm{HpHbR}$ receptor derived from $T . b$. brucei was genetically restored. Altogether, we identify two HpHbR-dependent evolutionary trade-offs for T. b. gambiense human infectivity.

\section{INTRODUCTION}

Through the combination of human infections and livestock trypanosomiasis, the neglected tropical diseases caused by African trypanosomes belonging to Trypanosoma brucei sensu lato (s.l.) have a significant impact on sub-Saharan rural development. Two subspecies of $T$. brucei proliferate in humans: T. brucei gambiense is responsible for a chronic and slowly progressing human trypanosomiasis, while Trypanosoma brucei rhodesiense causes an acute form of the disease (1). When injected by the blood feeding insect vector (tsetse fly; Glossina spp.) into the human tissue, animal-infecting T. brucei brucei is rapidly killed by a potent arm of the innate immune system, represented by trypanosome lytic factors (TLF) 1 and $2(2,3)$. TLF1 is composed of haptoglobin-related protein (4) and apolipoprotein L-1 (5) and targets the parasites efficiently by engaging the haptoglobinhemoglobin receptor $(\mathrm{HpHbR})$, the only invariant cell surface receptor known to date in kinetoplastid parasites $(6,7)$. 
The $\mathrm{HpHb}$ complex is formed by haptoglobin $(\mathrm{Hp})$ and extracellular hemoglobin $(\mathrm{Hb})$ resulting from intravascular hemolysis. The principal function of $\mathrm{HpHbR}$, which was named after its only ligand, is heme uptake (6). Indeed, same as other trypanosomes, T. brucei s.l. are heme auxotrophs (8), who acquire external heme via $\mathrm{HpHbR}$ in the mammalian-infective bloodstream stage (BS) (6). The procyclic trypanosome stage (PS) in the tsetse fly midgut contains more hemoproteins and obtains the ancient and omnipresent heme cofactor using another dedicated transporter, $\mathrm{TbHrg}(9)$.

Nevertheless, $\mathrm{HpHbR}$ is not essential for the proliferation of BS since the monomorphic T. b. brucei knock-out for $\mathrm{HpHbR}$ ( $\mathrm{HpHbR} \mathrm{KO)} \mathrm{shows} \mathrm{only} \mathrm{a} \mathrm{mildly} \mathrm{affected}$ growth phenotype in vitro $(6,10)$. Moreover, despite being more sensitive to the host's oxidative stress, this cell line kills its rodent host before the first wave of immunoglobulinbased immunity develops (6). Curiously, the capacity of T. b. gambiense to survive in human blood and cause infection is partially based on a point mutation in $\mathrm{HpHbR}$ that dramatically reduces its affinity for both TLF1 and $\mathrm{HpHb}(10,11,12,13)$.

Retaining the $\mathrm{HpHbR}$ expression contribute to trypanosome's fitness in their animal reservoir hosts, providing positive selection pressures for the conservation of this receptor $(6,14)$. Still, it acquired critical mutations that allowed T. b. gambiense to enter a new niche, the human host, despite the attenuation in the primary animal hosts. The cost of this loss of $\mathrm{HpHbR}$ function was tolerable, although it brought a reduced capacity for cyclical transmission by the tsetse fly when compared with the closely related T. b. brucei and T. $b$. rhodesiense.

Here we confirm that $T$. b. brucei $\mathrm{HpHbR}$ KO does not uptake heme and newly demonstrate that it leads to the loss of the ability to fuel hemoproteins with this cofactor. Moreover, the loss of this receptor is associated with a reduced ability to undergo differentiation in the mammalian host. In the absence of $\mathrm{HpHbR}$, the fast dividing long slender forms of the bloodstream stage (BS-SL) do not transform into the quiescent, transmission-competent stumpy forms of the bloodstream stage (BS-ST). As a natural mutant for $\mathrm{HpHbR}$, the human pathogenic $T$. b. gambiense is poorly capable of importing heme and generating BS-ST, while both key features are restored by heterologous expression of the $T$. b. brucei-derived HpHbR. 


\section{RESULTS}

\section{Heme-free trypanosomes}

T. brucei s.l. lost the capacity to synthesize heme and has to acquire it from its hosts (8). First, we verified previous fluorimetric measurements of heme amount (6) and showed consistent values from the mouse-collected BS parasites. Wild type (WT) T. b. brucei contains 100 pmol heme $/ 10^{9}$ cells, contrary to the $T$. b. brucei HpHbR KO cells, which exhibit only a trace amount of heme at the detection limit (Figs. 1A, B). For the first time, we established the amount of heme in WT T. b. gambiense, where also only a trace of heme was observed (6 pmol/109 cells), comparable to $\mathrm{HpHbR}$ KO cells (Figs. 1A, B).

Next, we studied how hemoproteins function under these conditions. Therefore, a V5-tagged human catalase (hCAT), a potent and widespread heme-dependent enzyme which is absent in the genome of T. brucei s. I. (15), was expressed in three cell lines, namely WT T. b. brucei and the derived HpHbR KO, and WT T. b. gambiense (Figs. 1C, D). This allowed us to follow the activity of exogenous hemoprotein, which showed a uniform distribution in the cytosol (Fig. 1E). The catalase activity was monitored via the production of molecular oxygen, forming visible bubbles in the cell suspension (Fig. 1F; inset) upon the addition of $\mathrm{H}_{2} \mathrm{O}_{2}$ as a substrate (Fig. $1 \mathrm{C}$ ). This assay showed that catalase was active only in WT T. b. brucei (Fig. 1F), while it was inactive in both T. b. brucei HpHbR KO and T. $b$. gambiense (Fig. 1F). This result is best explainable by the failure of the latter trypanosomes to import heme due to the absence of a functional $\mathrm{HpHbR}$. This experiment also demonstrated that when taken up by $\mathrm{HpHbR}$, heme is delivered into the cytosol, where it is freely available for hemoproteins as a cofactor.

\section{Endogenous hemoproteins activity depends on $\mathrm{HpHbR}$}

Being involved in the sterol metabolism $(16,17)$, the hemoprotein CYP51 is considered to be essential and, therefore, a promising drug target against different trypanosomatids $(18,19,20)$. Ketoconazole is one of the broadly used compounds which binds to the active site of CYP51 and inhibits its activity (21). The genome of T. brucei s.l. encodes a single copy of CYP51, which is transcribed primarily in the PS and only weakly in the BS-SL and BS-ST cells (Fig. 2A). RNAi in the PS led to an efficient reduction of CYP51 mRNA (Suppl. Fig. 1A) followed by almost complete elimination of the corresponding protein 5 days post-induction (Suppl. Figs. 1B), as also illustrated by reduced $\alpha$-CYP51 
immunostaining in the RNAi-induced cells (Suppl. Fig. 1C). The gradual loss of CYP51 was associated with a significant growth reduction of the PS cells (Suppl. Fig. 1D). Of note, we were unable to generate CYP51 KO cell lines in the PS suggesting its essentiality in the given stage.

In contrast to PS, CYP51 RNAi in the BS did not result in a significant growth alteration (Fig. 2B). However, the extent of mRNA down-regulation was not complete, leaving the basal level of expression (data not shown). Therefore, we generated CYP51 KO by homologous recombination of both alleles with hygromycin and phleomycin expression cassettes in the BS cells (Suppl. Fig. 1E). Again, the growth phenotype of the BS lacking CYP51 was only slightly affected, indicating that this protein is dispensable in this stage (Fig. 2C).

Next, we evaluated the sensitivity of CYP51 RNAi and KO cells to ketoconazole, a specific inhibitor of CYP51 (Figs. 2D, E). In agreement with the previously published IC 50 values (22), there was no statistical difference between the parental WT and CYP51 KO cells (Fig. 2D). Still, concentrations of ketoconazole ranging from 2 to $8 \mu \mathrm{M}$ affect the WT and CYP51 KO cells differentially, since the former cells reduced their growth rate, while the latter remained unaffected (note the biphasic behavior of the dose-response curve) (Fig. 2D; right panel). An identical effect was observed when the sensitivity to ketoconazole was followed in the CYP51 RNAi BS trypanosomes (Fig. 2D; left panel). Hence, CYP51 activity can be selectively inhibited with low doses of ketoconazole, discriminating between the cells with and without CYP51. In contrast, when higher doses of ketoconazole are applied, we do not see significant differences in the growth of WT and CYP51 mutant and the action of the drug should be assigned to the off-target effect.

\section{$\mathrm{HpHb}$ uptake deficiency confers insensitivity to CYP51 inhibition}

The pharmacological conditions described above allow assessing the activity of the hemoprotein CYP51 in the BS cells under the conditions of defective $\mathrm{HpHb}$ uptake. First, we exposed $\mathrm{HpHbR} \mathrm{KO}$ cells to ketoconazole and showed they are insensitive to low doses of the drug (Fig. 2F), mimicking the phenotype observed for CYP51 RNAi and KO cells.

Next, the HpHbR pathway-dependent CYP51 activity readout was evaluated in the WT T. b. rhodesiense cells resistant to the lysis by the human serum independently of $\mathrm{Hp}-\mathrm{Hb}$ uptake (23). Under different cultivation conditions, we modulate their access to the cognate ligand of the receptor. When grown in the Hp-containing human serum, in which the 
161

162

163

164

165

166

167

168

169

170

171

172

173

174

175

176

177

178

179

180

181

182

183

184

185

186

187

188

189

190

191

192

heterodimeric $\mathrm{HpHb}$ ligand is formed, $T$. $b$. rhodesiense is sensitive to micromolar concentrations of ketoconazole (Fig. 2G). In contrast, when grown in the anhaptoglobinemic serum $(24,25)$, i.e. in the absence of $\mathrm{Hp}$, cells exhibit reduced growth and become insensitive to the drug. Yet, complementation of the anhaptoglobinemic serum with purified human $\mathrm{Hp}$ reverted this phenotype (Fig. $2 \mathrm{G}$ ), proving a positive correlation between the operational $\mathrm{HpHb}$ uptake and the CYP51 activity.

Combined, by indirect means, we showed the CYP51, as one of the genuine hemoproteins in trypanosomes is the downstream acceptor of the $\mathrm{HpHb}$ complex.

\section{Artificial expression of HpHbR in stumpy forms does not interfere with life cycle progression}

Due to RNA polymerase I-mediated polycistronic transcription, in trypanosomes, most regulation occurs post-transcriptionally (26). It was shown previously that the $\mathrm{HpHbR}$ expression is downregulated in the early phase of the BS-SL to BS-ST differentiation (27). We noticed that $H p H b R$ and other genes involved in this interstagial switch are located at the very end of the polycistronic transcription units (data not shown). Therefore, we created a cell line ( $\mathrm{HpHbR}-\mathrm{Luc}$ ), where the distance of $\mathrm{HpHbR}$ from the end of the polycistron was artificially increased by the insertion of a $10 \mathrm{~kb}$-long Luciferase gene in front of the procyclin gene (Suppl. Fig. 2C). By following the expression of both $\mathrm{HpHbR}$ and Luciferase genes during in vitro differentiation, we detected that the Luciferase mRNA was continuously formed as judged by activity measurements (Fig. 3A). In contrast, the $H p H b R$ mRNA was downregulated in HpHbR-Luc to the same extent as in the WT cells during the PS to BS differentiation (Fig. $3 \mathrm{~B})$. This data suggest that the distance from the end of the polycistronic unit is not a critical factor orchestrating the transcription efficiency during life cycle progression.

It is well known that $3^{\prime}$ untranslated regions (UTRs) have a critical role in the regulation of transcripts stability in trypanosomes. We forced the expression of $H p H b R$ in the BS-ST by fusing the $H p H b R$ open reading frame to the 3' UTR of PAD1 gene (Suppl. Fig. 2D), the product of which is exclusively expressed in the BS-ST (28). In this HpHbR-3'PAD1 cell line, the $H p H b R$ expression is maintained until the in vitro differentiation reaches the PS, which expresses $5 x$ more $H p H b R$ mRNA when compared to the WT PS (Fig. 3C). The capacity of the HpHbR-3'PAD1 cell line to differentiate from the BS-SL to the BS-ST in vivo was evaluated in mice, where the typical parasitic wave characteristic for the WT trypanosomes 
was produced (Fig. 3D). Moreover, ex vivo cells collected on day 4 were examined morphologically and the functionality of $\mathrm{HpHbR}$ was verified by the uptake of the fluorescently-labeled HpHb complex (Figs. 3E, F). As expected, the WT cells produced around

196 75\% of the BS-ST cells, which were exclusively $\mathrm{Hp}$-free. The HpHbR-3'PAD1 cells also retained the ability to form the BS-ST cells ( $60 \%$ by day 4 ), all of which were $\mathrm{Hp}$-positive due to the artificial expression of the receptor (Figs. 3E, F).

Since the ability of the HpHbR-3'PAD1 cell line to differentiate has been tested only in the mammalian host, we decided to also evaluate its capacity for transmission via tsetse flies. The midguts of flies fed with blood containing either the WT or HpHbR-3'PAD1 BS parasites were dissected after 10 days post-feeding, showing no significant difference in the infection rates (Fig. 3G). Next, we added reduced L-glutathione to the blood meal, which is known to enhance the trypanosome establishment in the tsetse midgut (29). Indeed, the infection rate reached up to $90 \%$, but we did not observe a significant difference between the WT and HpHbR-3'PAD1 cells, and their ability to transform to the PS and establish the tsetse midgut infection (Fig. 3G). Altogether, these results show that trypanosomes with the prolonged expression of $\mathrm{HpHbR}$ still differentiate into the BS-ST and subsequent PS and retain the ability to infect tsetse flies.

\section{T. b. brucei HpHbR KO does not produce stumpy forms}

The BS trypanosomes undergo extensive cellular differentiation in preparation for an abrupt transmission from the mammalian blood into tsetse fly. As the intensity of infection increases through the rapid proliferation of the BS-SL cells, a parasite-derived stumpy induction factor accumulates, which promotes morphological transformation into the BS-ST (30).

In order to test whether heme and/or HpHbR play any role in this critical phase of the life cycle, the HpHbR KO has also been generated in the pleomorphic 90-13 T. b. brucei by replacing both alleles with the puromycin or phleomycin cassette (Suppl. Figs. 2A, B). Moreover, we have created an addback cell line, in which the HpHbR expression was restored from the $18 \mathrm{~S}$ rRNA locus (18S AB), while in another cell line, a single $\mathrm{HpHbR}$ allele was restored in situ (in situ $\mathrm{AB}$ ). First, the functionality of $\mathrm{HpHbR}$ in all tested cell lines was examined by fluorescence microscopy of $\mathrm{Hp}$-labeled with a green fluorochrome (Hp-Alexa 488). As expected, the uptake of the $\mathrm{HpHb}$ complex was disrupted entirely in the $\mathrm{HpHbR} \mathrm{KO}$ 
cell line, while $80 \%$ of the WT cells were Hp-positive (Figs. 4A, B). Both above-described addback cell lines showed the re-establishment of the Hp uptake, although to a different extent. Overexpression of $\mathrm{HpHbR}$ from the 18S rRNA locus almost reached the WT values (70\%), whereas only $20 \%$ of in situ AB cells were labeled (Figs. $4 A, B$ ). Next, the capacity to undergo differentiation in vitro was analyzed by exposing the individual cell lines to cis-aconitate and a temperature decrease to $27^{\circ} \mathrm{C}$, which is known to trigger the BS to PS transformation. We followed their ability to proliferate and express the procyclin coat as a hallmark of the PS (Figs. 4C, D). Under these conditions, the majority of the WT cells (70\%) became procyclin-positive by day 2 and reached high densities $\left(5 \times 10^{6}\right.$ cells/ml) by day 3 (Figs. 4C, D). In contrast, the HpHbR-KO cells differentiated into only a few PS cells that did not divide and eventually died out (Figs. 4C, D). Both AB cell lines showed a high capacity to differentiate in vitro, with 70\% of cells covered by procyclin by day 2 (Fig. 4D), although the cell numbers were about half when compared to the WT (Fig. 4C). experiments, since their genetic background is more physiologically relevant compared to the bulky HpHbR expression from the 18S rRNA locus. The selected cell lines were analyzed for their capacity to differentiate in vivo. As shown in mice, the HpHbR KO cells initially proliferate somewhat slower as compared with the WT. However, on day 6 the infection rapidly accelerated, achieving a high parasitemia of $\sim 3 \times 10^{8}$ cells $/ \mathrm{ml}$, leading to the termination of the experiment on the following day (Fig. 4E). In contrast, the WT (90-13) parasitemia declined on day 6 , while the in situ $A B$ cells went halfway, reaching a plateau on day 7 (Fig. 4E).

The blood-harvested parasites were further examined for the PAD1 expression using immunofluorescence microscopy and Western blot analysis (Figs. 4 F-I). Since the PAD1 protein is specifically expressed on the surface of the BS-ST and is prominently absent from the BS-SL, it is used as a molecular marker for the former morphotype (31). At the same time, we followed characteristic morphological features, such as the distance between the kinetoplast and the nucleus and the cell volume (Figs. 4F). An exhaustive analysis failed to detect PAD1 in the HpHbR KO trypanosomes (Figs. $4 \mathrm{H}, \mathrm{I}$ ), which is in line with their exclusive BS-SL morphology (Figs. 4F, G). On the contrary, 97\% of the WT cells were PAD1-positive (Figs. $4 \mathrm{H}, \mathrm{I}$ ) and had the characteristic BS-ST morphology, associated with larger cell volume and shorter distance between the kinetoplast and the nucleus (Fig. 4F). Additionally, the in 
situ AB cells also expressed the PAD1 protein, although to a lesser extent (30\%) (Figs. $4 \mathrm{H}, \mathrm{I}$ ), which was accompanied by the intermediate to the BS-ST phenotype (Fig. 4F).

\section{Restoration of stumpy formation in $T$. b. gambiense}

Next, we studied the consequences of a restored $\mathrm{HpHbR}$ expression in T. brucei gambiense for its life cycle progression in the mammalian host. We used the WT T. $b$. gambiense (LiTat1.3 strain; T.b.g. WT) and the T. b. gambiense cells expressing HpHbR of T. b. brucei from the $18 \mathrm{~S}$ rRNA locus (T.b.g. $+\mathrm{b} 1$ ), as described previously (13). Both cell lines were injected into the BALB/c mice and the resulting parasitemia was followed on a daily basis. The WT parasites emerged in the bloodstream between days 2 and 3 and sustained a rather mild infection (maximum of $3 \times 10^{7}$ cells $/ \mathrm{ml}$ was reached on day 4), as on day 6 no trypanosomes were observed in the blood smears (Fig. 5A). The T. b. gambiense expressing $\mathrm{HpHbR}$ of $T$. b. brucei triggered a yet significantly weaker infection, with cells detectable only until day 4 , when they peaked at $5 \times 10^{5}$ cells $/ \mathrm{ml}$ (Fig. 5A).

In the WT T. b. gambiense, we did not detect any PAD1-expressing cells, while the picture was strikingly different for trypanosomes, in which the T. b. brucei $\mathrm{HpHbR}$ was overexpressed. At the peak of the infection on day 4 , about $25 \%$ of cells were PAD1-positive (Figs. 5B, C), associated with a significant repositioning of the nucleus towards the kinetoplast (Fig. 5D). In contrast, there was no pronounced increase in the cell volume (Fig. 5D), a feature typical for the PAD1-expressing BS-ST T. b. brucei.

Finally, we wondered whether the T. b. gambiense field isolate Bosendja (32) sustains the ability to produce waves of parasitemia. Due to its limited viability in the axenic culture, Bosendja cells were injected directly from the blood stabilates into the BALB/c mice. The parasites proliferated very rapidly in their bloodstream, reaching over $3 \times 10^{8}$ cells $/ \mathrm{ml}$ on day 4, when the experiment was terminated (Fig. 5E). Careful morphological inspection categorized most trypanosomes into the BS-SL forms, with a pronounced undulating membrane (Fig. 5F). Although in a few cells (0.1\%) expression of PAD1 was detected on day 4 by immunomicroscopy (Figs. 5F, G), The low amount of this marker protein remained undetectable by Western blot analysis, where the WT T. b. brucei and the in situ AB cells served as positive controls (Fig. $5 \mathrm{H}$ ). 
The heme group is needed for the life of almost all eukaryotic organisms analyzed so far (33). Nevertheless, it has remained unclear whether the parasitic lifestyle of trypanosomes still needs this requirement while residing in the mammalian host. Indeed, many hemoproteins are missing or downregulated in the BS T. b. brucei. To provide heme for its hemoproteins, such as the subunits of respiratory complexes (and enzymes involved in the sterol synthesis (17), the BS and PS T. b. brucei scavenge heme from their hosts via the $\mathrm{HpHbR}$ and $\mathrm{TbHrg}$ receptors, respectively $(6,9)$.

The HpHbR of $T$. brucei s.l. has undergone a remarkable set of adaptations, likely triggered by coevolution with their distinct hosts. To avoid lysis by the innate immunity factors in the blood of primates, the $\mathrm{HpHbR}$ of $T$. $b$. gambiense acquired specific mutations that decreased the affinity towards its ligand $(10,11,13)$. Its lower affinity for TLF as compared to $\mathrm{HpHb}$ led to the proposal that the cells maintain the $\mathrm{HpHb}$ uptake while resisting lysis (12). The closely related $T$. congolense and $T$. vivax use $\mathrm{HpHbR}$ to take up $\mathrm{Hb}$ rather than $\mathrm{HpHb}$ from the digested blood (34). Moreover, while in T. brucei s. I. HpHbR is confined to the flagellar pocket, where it was shown to be down-regulated during the stumpy formation (27), it is present on the cell surface of the $T$. congolense epimastigotes (35) at a density $1000-$ fold higher than in the BS T. b. brucei (34).

Following confirmation of the lack of heme $b$ detected in the BS T. $b$. brucei devoid of $\mathrm{HpHbR}(6)$, here we have shown that WT BS T. b. gambiense also contains minimal if any heme. We imply that the BS trypanosomes can grow in limited amounts of heme, suggesting a lack of essentiality of this ancient cofactor in this life cycle stage. As we reported earlier, heme is also dispensable for the plant trypanosomatid parasite Phytomonas serpens. Cells that were grown without heme incorporated lanosterol into the membranes instead of ergosterol, overcoming the need for hemoprotein CYP51 (36). In this study, we focused on the hemoprotein CYP51 in T.brucei, where we have shown that CYP51 is not essential for BS parasites and represents an acceptor of the HpHbR-imported heme. We have further evaluated the HpHbR-dependent CYP51 inhibition in the WT T. $b$. rhodesiense by modulating its access to the cognate ligand of the receptor and proved a positive correlation between an operational $\mathrm{HpHb}$ uptake and the cytokinetic CYP51 activity. overexpressed human catalase (37) in the WT and HpHbR KO T. b. brucei, as well as in the 
WT T. b. gambiense. The data conclusively showed that in T. b. brucei BS HpHbR internalizes heme, which is subsequently incorporated into the cytosolic hemoprotein.

Under physiological conditions, human blood is low in free $\mathrm{Hb}$. Lysed erythrocytes release an excess of $\mathrm{Hb}$ that binds to $\mathrm{Hp}$ with a picomolar affinity and forms a complex internalized by macrophages (38). Trypanosomes imitate this process via their $\mathrm{HpHbR}$, which competes for the ligand (39). Impaired erythrocytes and their clearance eventually cause anemia, which represents the primary pathological hallmark of animal trypanosomiasis (40). Intriguingly, reports of severe infection-associated anemia are missing for human T. $b$. gambiense infections, which can be even asymptomatic (41).

The erythrocytes from mice infected with trypanosomes exhibit an enhanced osmotic fragility and altered fatty acid membrane composition (42), which are caused by both host immune response and parasite-derived factors (43). It was also proposed that during the acute phase of mice infection, $T$. b. brucei releases extracellular vesicles that fuse with erythrocytes and consequently increase their fragility and clearance (44). We propose that trypanosome infection related $\mathrm{Hb}$-release may be one of the factors modulating the course of parasitic waves and deserves a closer look.

One of the main processes which are associated with parasitemia control in the mammalian host is the transition from the dividing BS-SL to the quiescent BS-ST (45). Recently, several proteins and effector molecules involved in this complex process have been described $(30,46,47)$. The fact that in the absence of $\mathrm{HpHbR}$, the key BS-SL to BS-ST transition is disrupted, prompts us to suggest that heme uptake may be an additional player in this life cycle progression. This assumption is further backed by our observation that the WT T. b. gambiense has a poor capability to generate typical BS-ST. Importantly, a mere replacement of the $T$. b. gambiense $\mathrm{HpHbR}$ by its functional $T . b$. brucei variant restores the capacity to progress into this life cycle stage. The accelerated pathogenicity for mice of $T . b$. brucei devoid of $\mathrm{HpHbR}$ is likely caused by the fast division of the BS-SL and the failure to develop into the non-dividing BS-ST, which would otherwise lead to protracted progress of the infection.

$\mathrm{HpHbR}$ is known to be a gateway for the internalization of TLF, which results in the lysis of T. b. brucei by human serum (39). Here we show that in T. b. gambiense, this receptor also effectively prevents the import of heme with consequences for the heme-requiring cellular processes. While this would be lethal for a typical aerobic eukaryote that depends on 
external heme, trypanosomatids, such as the abovementioned Phytomonas have a unique capacity to tolerate complete heme deficiency (36).

Changes in $T$. $b$. gambiense $\mathrm{HpHbR}$ seems to have far-reaching consequences with decreased and eventually abrogated heme import. Heme dispensability may be associated with lower pathogenicity and possibly result in the chronic form of the disease. However, unless experimentally tested, this will remain speculation backed by the lower pathogenicity of the HpHbR KO T. b. brucei for both the Hp-carrying and Hp-lacking mice (6). Importantly, trypanosomes with defective HpHbR (T.b.b. HpHbR KO; T.b.g. WT) are struggling to progress into the BS-ST, which was so far considered the only stage of T. brucei s.l. capable of transmission to tsetse flies (47). However, this postulation has been challenged very recently (48), showing that T.b.b. BS-SL and BS-ST trypanosomes are equally capable of infecting tsetse flies. The transmissibility via tsetse fly of $T$. $b$. gambiense is known to be poor even under controlled experimental conditions, for so far unknown reasons (49, 50 , J. V. d. A., unpubl. data). To the best of our knowledge, the reporting of putative BS-ST cells in T. b. gambiense was based solely on morphological criteria $(51,52)$ and may have resulted in a misassignment to a different $T$. brucei sub-species that is more prone to BS-ST transition. More recent publications describe a high variability in the proportion of the BS-SL to BS-ST cells in different field strains of $T . b$. gambiense $(50,53)$. Moreover, no clear correlation was noted between the presence of the putative BS-ST and transmissibility via tsetse flies (53), further questioning the relevance of the BS-ST cells in T. b. gambiense.

In summary, we submit that the consequence of the unique metabolic independence on heme described above for T. b. gambiense, which accounts for a vast majority of human infections, resulted in the loss of transmission-competent PAD1-expressing BS-ST cells from their life cycle. This disadvantage may be compensated by lower pathogenicity and significantly prolonged chronic disease typical for T. b. gambiense-caused human African sleeping sickness.

\section{EXPERIMENTAL PROCEDURES}

\section{Cells growth and differentiation}

Bloodstream T. b. brucei 90-13 (328-114 single marker), T. b. rhodesiense EtTat1.2R and T. b. gambiense AnTat 1.3 were routinely cultivated at $37^{\circ} \mathrm{C}$ in HMI-9 medium (Thermo Fisher), supplemented with $20 \%$ heat-inactivated foetal bovine serum. Bloodstream T. b. brucei 427 
385

386

387

388

389

390

391

392

393

394

395

396

397

398

399

400

401

402

403

404

405

406

407

408

409

410

411

412

413

414

415

was grown in the same medium with $10 \%$ fetal bovine serum. Field isolate of $T$. $b$. gambiense (Bosendja; AnTAR 6; ZR/ KIN001) was kept by passaging in mice. Cell densities were measured using the $\mathrm{Z} 2$ Coulter counter (Beckman Coulter) or by hemocytometer.

\section{Transgenic cell lines}

Procyclic T. b. brucei 29-13 CYP51 RNAi and HpHbR-KO (328-114) cell lines were described previously $(6,54)$. T. b. brucei CYP51-KO (328-114) and 90-13 HpHbR-KO cell lines were generated by successive deletion of alleles with pPuro-KO or pHygro-KO, and pPhleo-KO constructs (13). The pTSArib HpHbR construct used to complement the 90-13 HpHbR-KO cell line was obtained by subcloning of $\mathrm{HpHbR}$ ORF into pTSARib Ble (blasticidin resistance) plasmid (23). The other constructs designed to modulate the level of $\mathrm{HpHbR}$ expression were generated from the pET-in situ construct (55) after the replacement of the hygromycin resistance gene by Ble. PCR-amplified DNA fragments were assembled and cloned into pETin situ Ble plasmid by recombination (InFusion, Clontech). The constructs are depicted in Suppl. Fig. 2. Trypanosomes were transfected with linearized plasmid DNA or with gelpurified PCR products as described elsewhere (56). Selection markers were applied 6 hours post-transfection, and clones were generated by limited dilution.

\section{Real-time PCR, Western blot analysis and Luciferase activity measurement}

Total RNA was extracted using Trizol reagent, and the cDNA was synthesized using the PrimeScript ${ }^{\mathrm{TM}} \mathrm{RT}$ Reagent Kit with gDNA Eraser (Takara) as described by the manufacturer with an oligo dT used instead of random primers. The qRT-PCR was performed with the CDNA using the SYBR-green stain (Takara). Primers used for $\mathrm{HpHbR}$ gene were FW 5'CCAATGTCGAGGTCGCCATGGCTGAGGGTTTAAAAACCAAAGACGAAG 3' and RV 5'CCAATGTGCGGCCGCAAGCTTGGCATAACTGCGGAAACCACTAACCAC 3'. The C1 primers (FW 5'TTGTGACGACGAGAGCAAAC 3' and RV 5'GAAGTGGTTGAACGCCAAAT 3') were used as endogenous control (57).

In order to detect protein expression in the bloodstream stage, lysates from $5 \times 10^{6}$ cells were separated on a $12 \%$ SDS-polyacrylamide gel, transferred to a PVDF membrane and probed with the monoclonal anti-V5 and anti- $\alpha$ tubulin antibodies (Thermo Fisher) at 1:2,000 and 1:5,000 dilutions, respectively. Alternatively, the samples were prepared the same way 
416 (except the lysates were not boiled but heated to $37^{\circ} \mathrm{C}$ ) and probed with anti-PAD1 and

417 anti-enolase antibodies at 1:1,000 and 1: 10,000 dilutions, respectively.

$4182 \times 10^{6}$ parasites were centrifuged, lysed and labeled by the Luciferase Assay kit as

419 recommended by the manufacturer (Promega). The readout was performed for 10 seconds

420 with Luminoskan TL Plus instrument (Labsystems).

421

422 Overexpression and activity assay for catalase

423 The construct for expression of human catalase (hCAT, NCBI gene ID NP_001743.1) was

424 modified from the one described previously (37) to be constitutively expressed. The

425 obtained constructs were linearized by Notl, electroporated into the bloodstreams of T. $b$.

426 brucei and T.b.gambiense and selected using $1 \mu \mathrm{g} / \mathrm{ml}$ hygromycin (Thermo Fisher).

427 The activity of catalase was monitored by respirometry using Oxygraph-2K (Oroboros) as an

428 amount of $\mathrm{O}_{2}$ produced after the addition of $\mathrm{H}_{2} \mathrm{O}_{2}$ and analyzed using the Oroboros DatLab

429 Software. T. b. brucei 90-13 and T. b. gambiense LiTat 1.3 WT were used to establish the

430 background of the respirometry experiment. Briefly, $1 \times 10^{6}$ of bloodstream cells

431 resuspended in $2 \mathrm{ml}$ of $\mathrm{HMI}-9$ medium were treated with $20 \mu \mathrm{l}$ of $882 \mathrm{mM} \mathrm{(3 \% )} \mathrm{H}_{2} \mathrm{O}_{2}$.

432 Alternatively, $5 \times 10^{6}$ parasites were resuspended in $10 \mu \mathrm{l}$ phosphate buffered saline (PBS)

433 and placed on a microscopic slide. The same volume of $3 \% \mathrm{H}_{2} \mathrm{O}_{2}$ was added to the cells,

434 mixed, and the formation of oxygen visible as macroscopic bubbles was monitored as a

435 readout for the catalase activity. All measurements and statistics were calculated from three

436 independent biological replicates.

437

438 Indirect immunofluorescence assay

439 For immunofluorescence analysis, $1 \times 10^{6}$ to $1 \times 10^{7}$ cells were fixed with $4 \%$

440 paraformaldehyde and settled on microscopic slides. After $10 \mathrm{~min}$ incubation at room

441 temperature, they were washed with PBS and permeabilized with $100 \%$ ice-cold methanol

442 for 20 min. Cells were incubated with 5\% fat-free milk in PBS-Tween (0.05\%) for $1 \mathrm{~h}$,

443 followed by incubation with primary anti-V5, anti-PAD1 or anti-CYP51 at 1:1,000 dilutions

444 and secondary Alexa Fluor-488 or Alexa Fluor-555 anti-rabbit IgG antibodies (Thermo Fisher)

445 at 1:1,000 dilution for $1 \mathrm{hr}$ at room temperature. After the last washing step, cells were

446 stained with DAPI, mounted with an anti-fade reagent (Thermo Fisher) and visualized using a

447 fluorescent microscope Zeiss Axioplan 2 (Carl Zeiss). 
A total of $5 \times 10^{8}$ bloodstream cells was harvested by centrifugation at $1,000 \mathrm{~g}$ at $4^{\circ} \mathrm{C}$ for 10

450

451

452

453

454

455

456

457

458

459

460

461

462

463

464

465

466

467

468

469

470

471

472

473

474

475

476

477

478

min and washed 3 times with PBS on ice. Cells were resuspended in $60 \mu \mathrm{l} \mathrm{H}_{2} \mathrm{O}$, extracted with $400 \mu$ lacetone $/ 0.2 \% \mathrm{HCl}$, and the supernatant was collected after centrifugation at $1,000 \mathrm{~g}$ at $4^{\circ} \mathrm{C}$ for $5 \mathrm{~min}$. The pellet was resuspended in $200 \mu \mathrm{l}$ acetone $/ 0.2 \% \mathrm{HCl}$ and centrifuged as described above. Both supernatants were combined, and $150 \mu$ l of each sample was immediately injected into a high-performance liquid chromatography system (Infinity 1200, Agilent Technologies) and separated using a reverse-phase column (4 $\mu \mathrm{m}$ particle size, $3.9 \times 75 \mathrm{~mm}$ ) (Waters) with $0.1 \%$ trifluoroacetic acid and acetonitrile/0.1\% trifluoroacetic acid as solvents $\mathrm{A}$ and $\mathrm{B}$, respectively. Heme $b$ was eluted with a linear gradient of solvent $B(30-100 \%$ in $12 \mathrm{~min}$ ) followed by $100 \%$ of $B$ at a flow rate of $0.8 \mathrm{ml} / \mathrm{min}$ at $40^{\circ} \mathrm{C}$. Heme was detected by diode array detector (Infinity 1200, Agilent Technologies) and identified by retention time and absorbance spectra according to commercially available standard (Sigma-Aldrich).

\section{Fly and mouse infections}

Teneral tsetse flies were fed, 24-48 hours after emergence, with T. b. brucei Antat 1.1 WT and HpHbR-3'PAD1 strains infected blood meal, either or not supplemented with $10 \mathrm{mM}$ reduced L-glutathione to increase infection establishment. Parasites were harvested from the blood of cyclophosphamide-immunosuppressed mice (Endoxan) at 6-7 days postinfection and mixed with defibrinated horse blood (E\&O Laboratories). Flies were further fed every 2-3 days on uninfected defibrinated horse blood. Then, flies were dissected on day 10 after the first blood meal to assess the presence of parasites in the midgut (i.e. establishment of a PS midgut infection)

Four to six-week-old BALB/c mice were intraperitoneally injected with $1 \times 10^{4}$ (T.b. brucei strains) or $3 \times 10^{6}$ (T.b. gambiense strains) bloodstream cells. The infection was followed daily by diluting tail snip blood in TrypFix buffer (3.7\% formaldehyde, $1 \times$ SSC buffer) and manual counting of trypanosomes in a Neubauer hemocytometer. Mice were euthanized for the collection of parasites, which were separated from the erythrocytes on a diethylaminoethyl (DEAE) cellulose column using a standard protocol. Purified trypanosomes were washed once with PBS and subsequently used for downstream experiments. 


\section{Hp-488 preparation and labeling}

Hp was conjugated with Alexa 488 using the Dylight amine-reactive kit (Thermo Fischer) as recommended by the manufacturer. The blood from mice was collected at different times of infection from a tail puncture with a capillary containing heparin and centrifuged at 12,000 $\mathrm{rpm}$ for $3 \mathrm{~min}$ to separate the parasites, which were subsequently incubated at $37^{\circ} \mathrm{C}$ for 15 min in HMI-9 medium containing the lysosomal protease inhibitor FMK-024. The parasites were incubated in $20 \mathrm{\mu g} / \mathrm{ml}$ (f. c.) Alexa 488-labeled Hp for 2 hrs, subsequently fixed with 4\% paraformaldehyde for $10 \mathrm{~min}$, stained with DAPI and analyzed with a Zeiss Axioplan 2 epifluorescence microscope equipped with a Zeiss AxioCam HRm digital camera (Carl Zeiss, Thornwood, NY). Resulting images were analyzed using Adobe Photoshop software and Fiji.

\section{Ethics statement}

In Czech Republic, the research was approved by the Central Commission for Animal Welfare, Biology Centre (protocol No. 28/2016). All experimental procedures complied with the Czech law (Act No. 246/1992). In Belgium, the research was approved by the animal ethics committee of the Institute for Molecular Biology and Medicine and the Institute of Tropical Medicine (tsetse fly infection experiment). All mice were housed in a pathogen-free facility and the experiments were performed in compliance with the relevant laws and institutional guidelines (license LA1500474).

\section{REFERENCES}

1. Franco, J.R., Simarro, P.P., Diarra, A. \& Jannin, J.G. Epidemiology of human African trypanosomiasis. Clin. Epidemiol. 6, 257-75 (2014).

2. Hajduk, S.L. et al. Lysis of Trypanosoma brucei by a toxic subspecies of human highdensity lipoprotein. J. Biol. Chem. 264, 5210-5217 (1989).

3. Raper, J., Portela, M.P., Lugli, E., Frevert, U. \& Tomlinson, S. Trypanosome lytic factors: novel mediators of human innate immunity. Curr. Opin. Microbiol. 4, 402408 (2001).

4. Smith, A.B., Esko, J.D. \& Hajduk, S.L. Killing of trypanosomes by the human haptoglobin-related protein. Science 268, 284-286 (1995). 
5. Vanhamme, L. et al. Apolipoprotein L-I is the trypanosome lytic factor of human serum. Nature 422, 83-87 (2003).

6. Vanhollebeke, B. et al. A haptoglobin-hemoglobin receptor conveys innate immunity to Trypanosoma brucei in humans. Science 320, 677-681 (2008).

7. Stødkilde, K., Torvund-Jensen, M., Moestrup, S.K. \& Andersen, C.B. Structural basis for trypanosomal haem acquisition and susceptibility to the host innate immune system. Nat. Commun. 5, 5487 (2014).

8. Kořený, L., Lukeš, J., \& Oborník, M. Evolution of the haem synthetic pathway in kinetoplastid flagellates: an essential pathway that is not essential after all? Int. J. Parasitol. 40, 149-156 (2010).

9. Horáková, E. et al. The Trypanosoma brucei TbHrg protein is a heme transporter involved in regulation of stage-specific morphological transitions. J. Biol. Chem. 292, 6998-7010 (2017).

10. DeJesus, E., Kieft, R., Albright, B., Stephens, N.A. \& Hajduk, S.L. Single amino acid substitution in the group 1 Trypanosoma brucei gambiense haptoglobin-hemoglobin receptor abolishes TLF-1 binding. PLoS Pathog. 9, e1003317 (2013).

11. Symula, R.E. et al. Trypanosoma brucei gambiense group 1 is distinguished by a unique amino acid substitution in the $\mathrm{HpHb}$ receptor implicated in human serum resistance. PLoS Negl. Trop. Dis. 6, e1728 (2012).

12. Higgins, M.K. et al. Structure of the trypanosome haptoglobin hemoglobin receptor and implications for nutrient uptake and innate immunity. Proc. Natl Acad. Sci. USA 110, 1905-1910 (2013).

13. Uzureau, P. et al. Mechanism of Trypanosoma brucei gambiense resistance to human serum. Nature 501, 430-434 (2013).

14. Higgins, M.K., Lane-Serff, H., MacGregor, P. \& Carrington M. A. Receptor's tale: An eon in the life of a trypanosome receptor. PLoS Pathog. 26, 13:e1006055 (2017).

15. Kraeva, N. et al. Catalase in Leishmaniinae: With me or against me? Infect. Genet. Evol. 50, 121-127 (2017).

16. Zhou, W., Cross, G.A. \& Nes, W.D. Cholesterol import fails to prevent catalyst-based inhibition of ergosterol synthesis and cell proliferation of Trypanosoma brucei. J. Lipid. Res. 48, 665-673 (2007).

17. Nes, C.R. et al. Novel sterol metabolic network of Trypanosoma brucei procyclic and bloodstream forms. Biochem. J. 443, 267-277 (2012). 
18. Kessler, R.L., Soares, M.J., Probst, C.M. \& Krieger M.A. Trypanosoma cruzi response to sterol biosynthesis inhibitors: morphophysiological alterations leading to cell death. PLOS ONE 8, e55497 (2013).

19. McCall, L.I. et al. Targeting ergosterol biosynthesis in Leishmania donovani: essentiality of sterol 14alpha-demethylase. PLoS Negl. Trop. Dis. 9, e0003588 (2015).

20. Dauchy, F.A. et al. Trypanosoma brucei CYP51: essentiality and targeting therapy in an experimental model. PLoS Negl. Trop. Dis. 10, e0005125 (2016).

21. Zhang, J. et al. The Fungal CYP51s: Their functions, structures, related drug resistance and inhibitors. Front. Microbiol. 10, 691 (2019).

22. Lepesheva, G.I. et al. Sterol 14alpha-demethylase as a potential target for antitrypanosomal therapy: enzyme inhibition and parasite cell growth. Chem. Biol. 14, 1283-1293 (2007).

23. Lecordier. L. et al. Adaptation of Trypanosoma rhodesiense to hypohaptoglobinaemic serum requires transcription of the APOL1 resistance gene in a RNA polymerase I locus. Mol. Microbiol. 97, 397-407 (2015).

24. Koda, Y., Soejima, M., Yoshioka, N. \& Kimura, H. The haptoglobin-gene deletion responsible for anhaptoglobinemia. Am J Hum Genet. 62, 245-252 (1998).

25. Vanhollebeke, B. et al. Distinct roles of haptoglobin-related protein and apolipoprotein L-I in trypanolysis by human serum. Proc. Natl. Acad. Sci. USA 104, 4118-4123 (2007).

26. Clayton, C. Regulation of gene expression in trypanosomatids: living with polycistronic transcription. Open Biol. 9, 190072 (2019).

27. Vanhollebeke, B., Uzureau, P., Monteyne, D., Pérez-Morga, D. \& Pays, E. Cellular and molecular remodeling of the endocytic pathway during differentiation of Trypanosoma brucei bloodstream forms. Eukaryot. Cell 9,1272-1282 (2010).

28. MacGregor, P. \& Matthews, K.R. Identification of the regulatory elements controlling the transmission stage-specific gene expression of PAD1 in Trypanosoma brucei. Nucleic Acids Res. 40(16), 7705-7717 (2012).

29. MacLeod, E.T., Maudlin, I., Darby, A.C. \& Welburn, S.C. Antioxidants promote establishment of trypanosome infections in tsetse. Parasitology 134, 827-831 (2007).

30. Rojas, F. et al. Oligopeptide signaling through TbGPR89 drives trypanosome quorum sensing. Cell 176, 306-317 (2019). 
31. Silvester, E., McWilliam, K.R. \& Matthews, K.R. The cytological events and molecular control of life cycle development of Trypanosoma brucei in the mammalian bloodstream. Pathogens 6, 29 (2017).

32. Fontaine, F. et al. APOLs with low pH dependence can kill all African trypanosomes. Nat. Microbiol. 2, 1500-1506 (2017).

33. Hamza, I. \& Dailey, H.A. One ring to rule them all: Trafficking of heme and heme synthesis intermediates in the metazoans. Biochim. Biophys. Acta 1823, 1617-1632 (2012).

34. Lane-Serff, H. et al. Evolutionary diversification of the trypanosome haptoglobinhaemoglobin receptor from an ancestral haemoglobin receptor. eLife 5, e13044 (2016).

35. Eyford, B.A. et al. Differential protein expression throughout the life cycle of Trypanosoma congolense, a major parasite of cattle in Africa. Mol. Biochem. Parasitol. 177, 116-125 (2011).

36. Kořený, L. et al. Aerobic kinetoplastid flagellate Phytomonas does not require heme for viability. Proc. Natl. Acad. Sci. USA 109, 3808-3813 (2012).

37. Horáková, E. et al. Catalase compromises the development of the insect and mammalian stages of Trypanosoma brucei. FEBS J. 287, 964-977 (2020).

38. Kristiansen, M. et al. Identification of the hemoglobin scavenger receptor. Nature 409, 198-201 (2001).

39. Vanhollebeke, B. \& Pays, E. The trypanolytic factor of human serum: many ways to enter the parasite, a single way to kill. Mol. Microbiol. 76, 806-814 (2010).

40. Radwanska, M., Vereecke, N., Deleeuw, V., Pinto, J. \& Magez, S. Salivarian trypanosomosis: A review of parasites involved, their global distribution and their interaction with the innate and adaptive mammalian host immune system. Front. Immunol., 9, 2253 (2018).

41. Büscher, P. et al. Do cryptic reservoirs threaten gambiense-sleeping sickness elimination? Trends Parasitol. 34, 197-207 (2018).

42. Cnops, J. et al. NK-, NKT- and CD8-derived IFNy drives myeloid cell activation and erythrophagocytosis, resulting in trypanosomosis-associated acute anemia. PLOS Pathog. 11, e1004964 (2015).

43. Stijlemans, B., de Baetselier, P., Magez, S., van Ginderachter, J.A. \& De Trez, C. African trypanosomiasis-associated anemia: the contribution of the interplay between parasites and the mononuclear phagocyte system. Front Immunol. 9, 218 (2018). 
44. Szempruch, A.J. et al. Extracellular vesicles from Trypanosoma brucei mediate virulence factor transfer and cause host anemia. Cell 164, 246-257 (2016).

655

656

657

658

659

660

661

662

663

664

665

666

667

668

669

670

671

672

673

674

675

676

677

678

679

680

681

682

683

684

685

686

687

688

689

690

691

692

693

694

695

696

697

698

45. Seed, J.R. \& Wenck, M.A. Role of the long slender to short stumpy transition in the life cycle of the African trypanosomes. Kinetopl. Biol. Dis. 2, 3 (2003).

46. Mony, B.M. et al. Genome-wide dissection of the quorum sensing signalling pathway in Trypanosoma brucei. Nature 505, 681-5 (2014).

47. Rojas, F. \& Matthews, K.R. Quorum sensing in African trypanosomes. Curr. Opin. Microbiol. 52, 124-129 (2019).

48. Schuster, S. et al. A modification to the life cycle of the parasite Trypanosoma brucei. bioRxiv717975; doi: https://doi.org/10.1101/717975 (2019).

49. Le Ray, D. Vector susceptibility to African trypanosomes. Ann. Soc. Belg. Med. Trop. 69, 165-214 (1989).

50. Ravel, S., Patrel, D., Koffi, M., Jamonneau, V. \& Cuny, G. Cyclical transmission of Trypanosoma brucei gambiense in Glossina palpalis gambiensis displays great differences among field isolates. Acta Trop. 100, 151-155.

51. Wijers, D.J., \& Willett, K.C. (1960). Factors that may influence the infection rate of Glossina palpalis with Trypanosoma gambiense. II. The number and morphology of the trypanosomes present in the blood of the host at the time of the infected feed. Ann. Trop. Med. Parasitol. 54, 341-350 (2006).

52. Brickman, M.J. \& Balber, A.E. Trypanosoma brucei brucei and T. b. gambiense: stumpy bloodstream forms express more CB1 epitope in endosomes and lysosomes than slender forms. J Eukaryot. Microbiol. 41, 533-536 (1994).

53. Janelle, J. et al. Monitoring the pleomorphism of Trypanosoma brucei gambiense isolates in mouse: impact on its transmissibility to Glossina palpalis gambiensis. Infect. Genet. Evol. 9, 1260-1264 (2009).

54. Haubrich, B.A. et al. Discovery of an ergosterol-signaling factor that regulates Trypanosoma brucei growth. J. Lip. Res. 56, 331-341 (2015).

55. Kelly, S. et al. Functional genomics in Trypanosoma brucei: a collection of vectors for the expression of tagged proteins from endogenous and ectopic gene loci. Mol Biochem Parasitol. 154(1),103-109 (2007).

56. Burkard, G., Fragoso, C.M. \& Roditi, I. Highly efficient stable transformation of bloodstream forms of Trypanosoma brucei. Mol. Biochem. Parasitol. 153, 220-223 (2007). 
710

711

712 Conceptualization, B.V., E.H.; Methodology, E.H., L.L., R.S., B.V.; Investigation, E.H., L.L.,

713 P.Cunha., R.S., P. Changmai., C.J.M.L., J.V.D.A., B.V.; Resources, B.V. and J.L.; Writing, E.H., 714 L.L., P.Cunha, J.V.D.A., B.V. and J.L.

715

716

717

57. Jones, N.G. et al. Regulators of Trypanosoma brucei cell cycle progression and differentiation identified using a kinome-wide RNAi screen. PLoS Pathog. 10, e1003886 (2014).

\section{ACKNOWLEDGEMENTS}

We thank Eva Kriegová and Zuzana Vavrušková (Institute of Parasitology) for help with animal experiments. We thank Nick Van Reet (Institute of Tropical Medicine, Antwerp) for providing the field strains of T.b. gambiense. This work was supported by the ERC CZ project LL1601 to J.L., ERD Funds project OPVVV 0000759, and Czech Grant Agency projects 1815962S and 20-07186S.

\section{AUTHOR CONTRIBUTIONS}

\section{DECLARATION OF INTERESTS}

The authors declare no competing interests. 
a
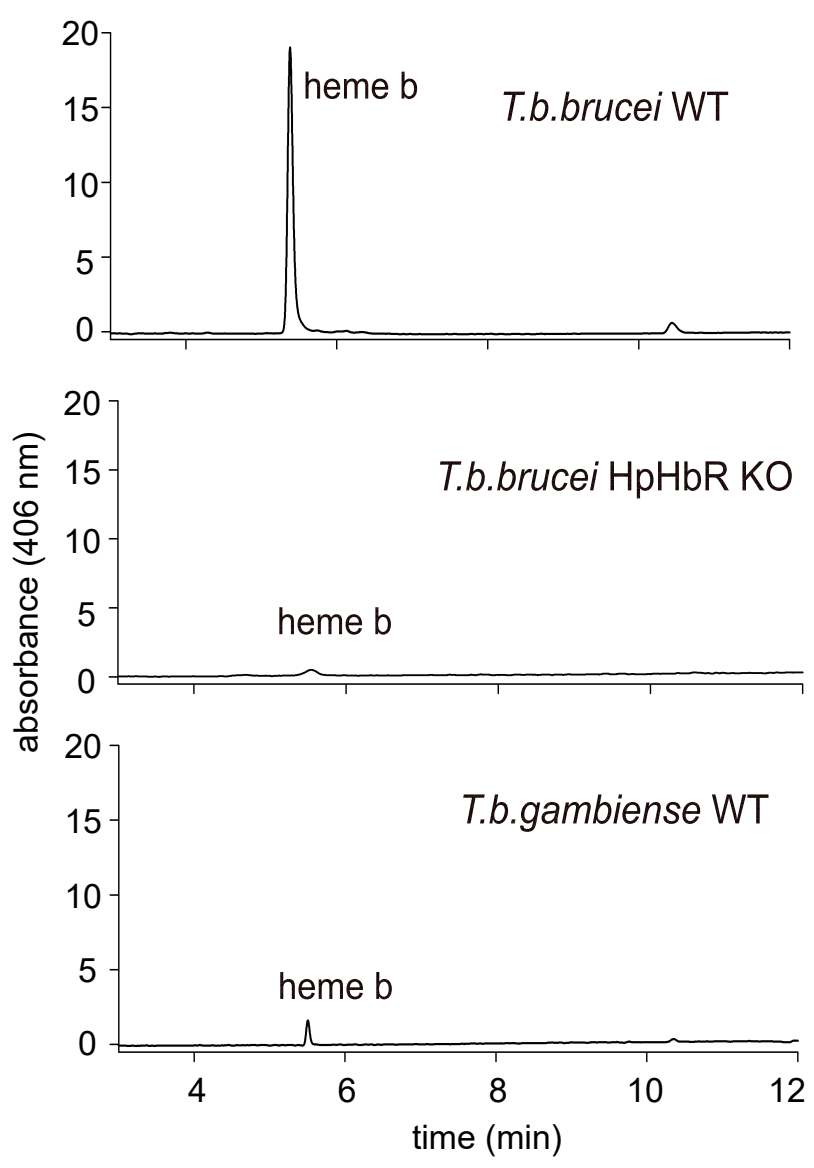

$\mathbf{f}$

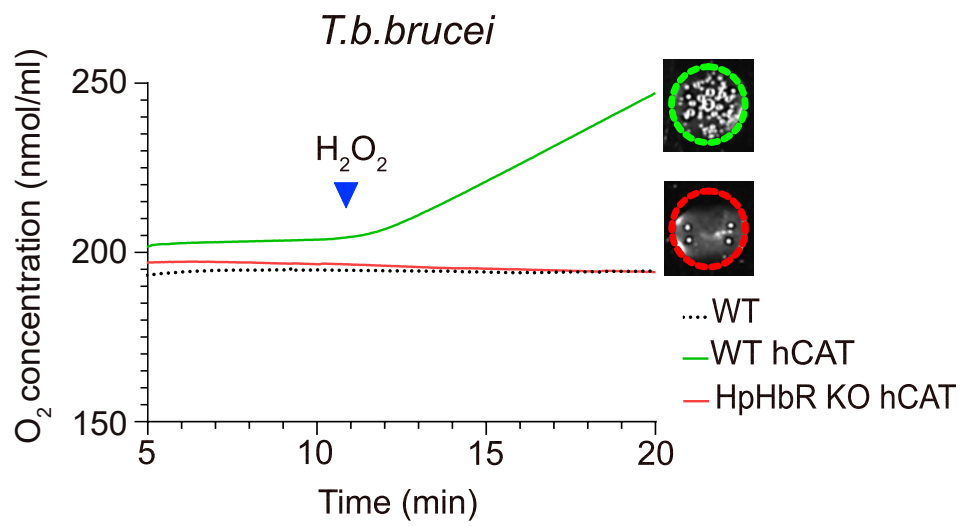

b

d

e
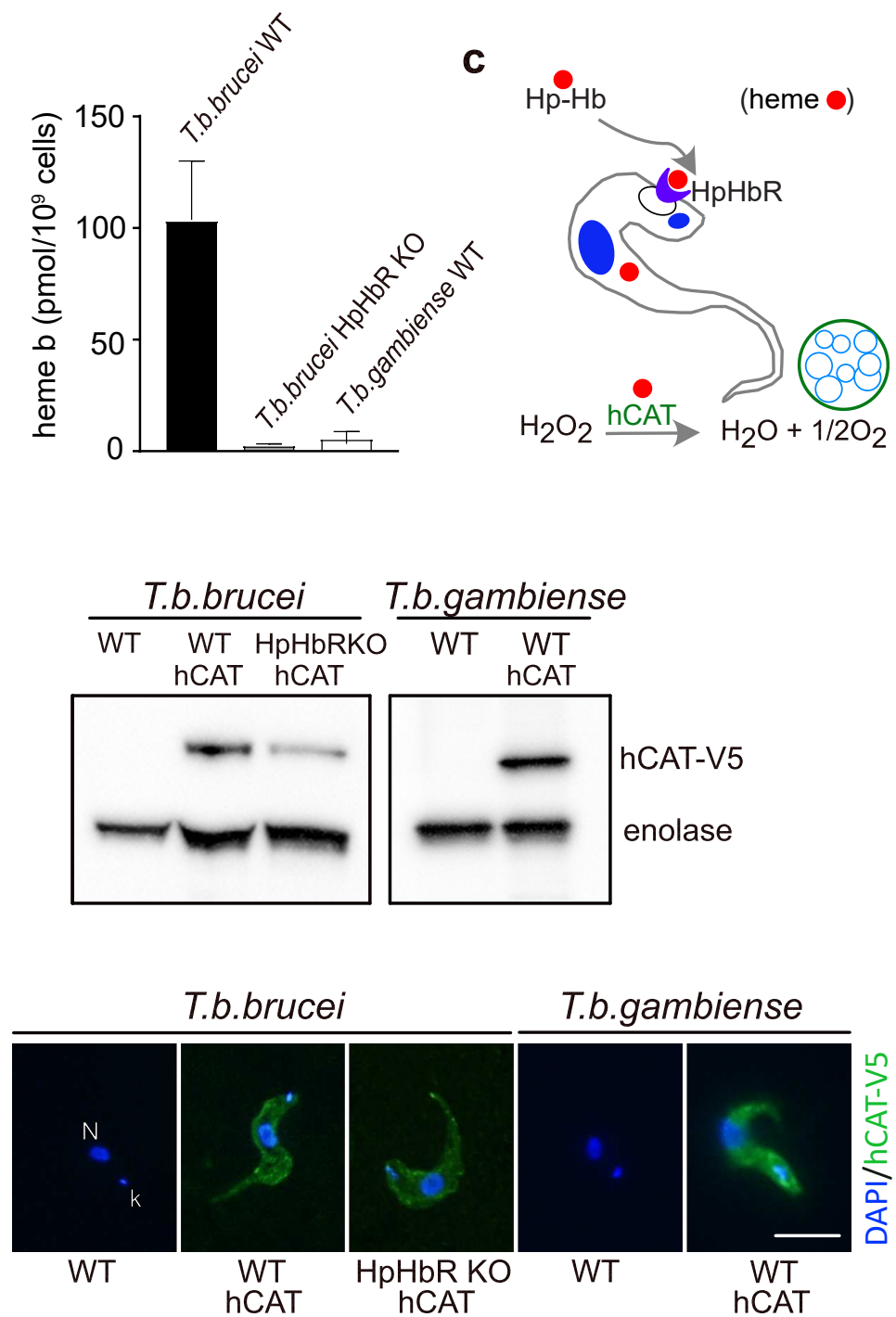

T.b.gambiense

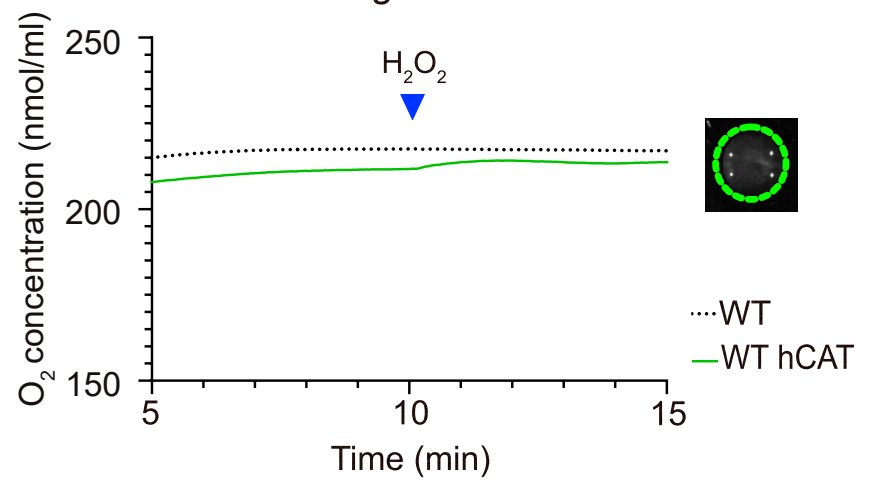

Figure 1. Detection of heme and hemoproteins in bloodstream stages of $T$. b. brucei and T. b. gambiense

(a) Heme $b$ extracted from $1 \times 10^{9}$ cells was separated by HPLC and detected by a diode array detector.

Representative chromatogram from wild type T. b. brucei, T. b. brucei knock-out for HpHbR and wild type T. b. gambiense. (b) Graph showing quantification of total heme $b$ content in the same cell lines as in $(a) ;(n=3)$. (c) Schematic representation of the experimental design used for the measurement of the activity of the N-terminally V5-tagged human catalase (hCAT) in bloodstream T. brucei. (d) Western blot analysis with V5 antibody that detects human catalase (hCAT) in WT and $\mathrm{HpHbR}$ knock-out (KO) of T. b. brucei and WT T. b. gambiense overexpressing hCAT. Enolase was used as a loading control. (e) Immunofluorescence of hCAT detected with V5 antibody (green) in the same cell lines as in (d). DNA in the nucleus $(\mathrm{N})$ and kinetoplast $(\mathrm{k})$ was stained with DAPI (blue). Scale bar, $5 \mu \mathrm{m}$. (f) Measurement of the activity of human catalase using Oroboros oxygraph in T. b. brucei (left) and T. b. gambiense (right); $(n=3)$. Visual verification of measured activity via the $\mathrm{O}_{2}$ production in the form of bubbles after the addition of $3 \% \mathrm{H}_{2} \mathrm{O}_{2}$ is shown in insets. 
a

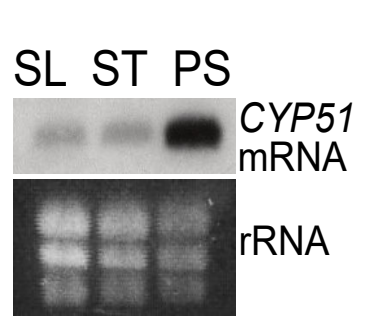

b

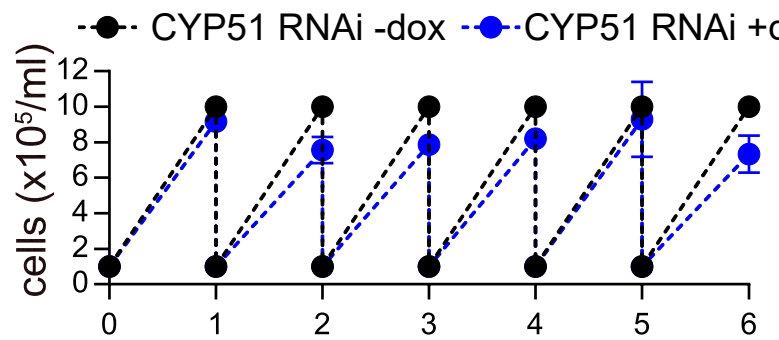

days
C

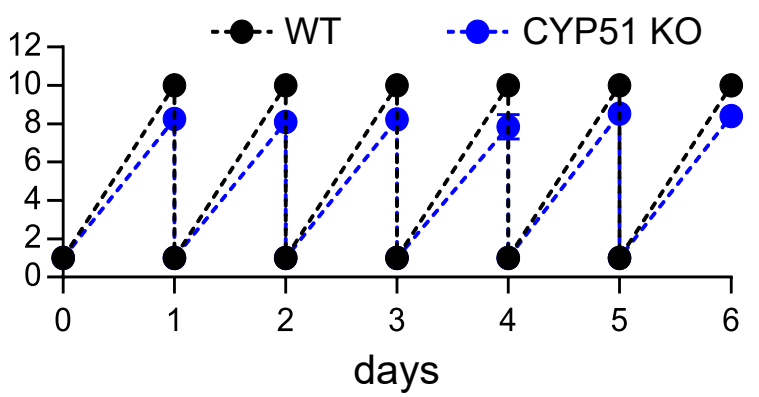

e

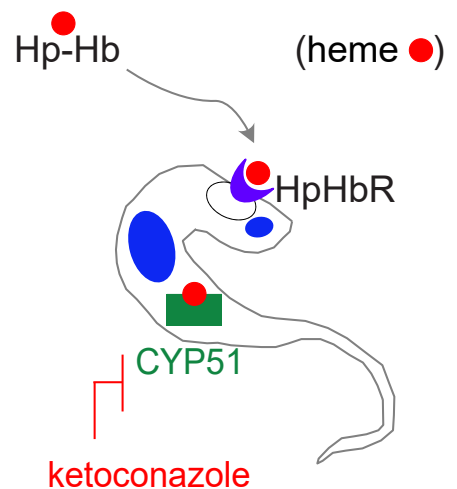

ketoconazole

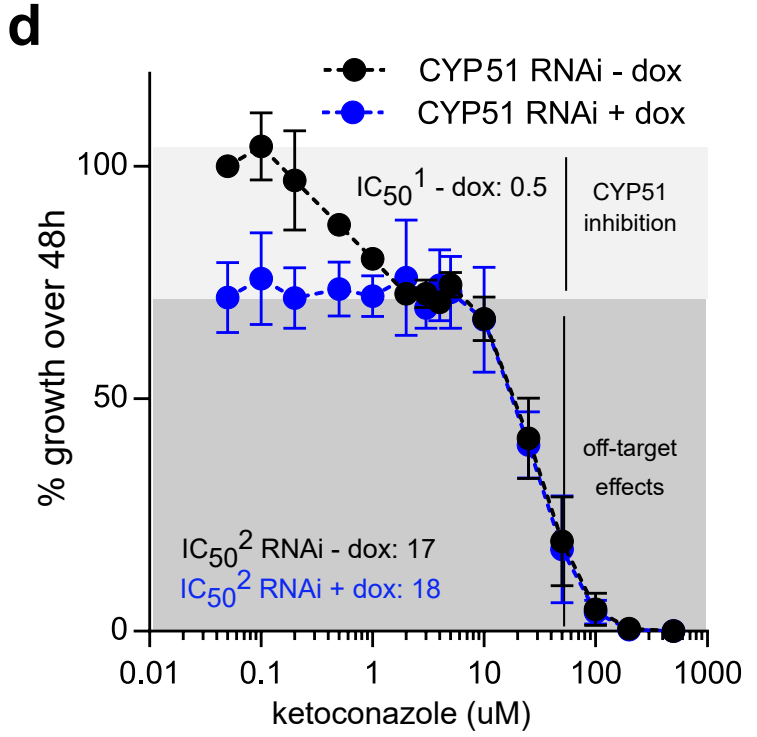

f

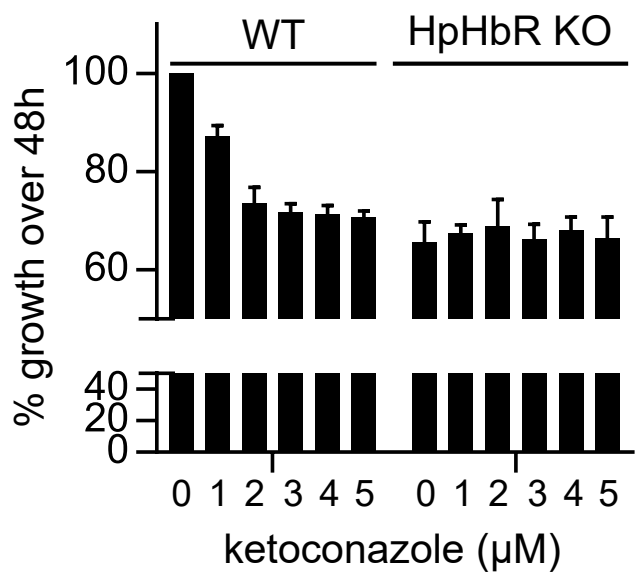

g

T.b. rhodesiense

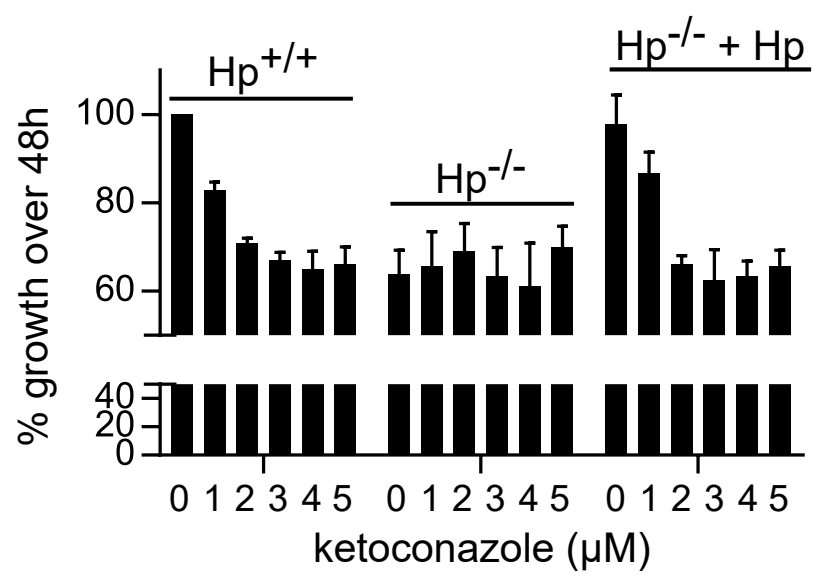


Figure 2. Effect on growth and ketoconazole sensitivity after CYP51 invalidation in the bloodstream stage

(a) Northern blot analysis showing stage-specific expression of CYP51 mRNA. Total RNA from long slender (SL) and stumpy (ST) bloodstream stage, and procyclic stage (PS) was hybridized with the TbCYP51 DNA probe. The staining of ribosomal (r)RNA was used as a loading control. (b) Growth curves of T. b. brucei bloodstreams in the presence (blue dots and line) and absence (black dots and line) of doxycycline (dox), which induces CYP51 RNAi for 6 days. (c) Growth curves of WT T. b. brucei (black dots and line) and CYP51 knock-out (KO) (blue dots and line) for 6 days. (d) The depletion of CYP51 confers insensitivity to low doses of ketoconazole. Left: CYP51 RNAi cells were grown with doxycycline to induce RNAi (blue dots and line) or without it (non-induced cells; black dots and line), during $48 \mathrm{~h}$ exposure to ketoconazole. Right: WT (black dots and line) and CYP51 knock-out (KO) cells (blue dots and line) were grown under the same conditions. A grey zone indicates concentrations of ketoconazole without inhibitory effect on CYP51. (e) Schematic representation of the experimental design showing the inhibition of hemoprotein CYP51 activity by ketoconazole. (f) Deficiency in the haptoglobin-hemoglobin $(\mathrm{HpHb})$ uptake results in mild growth phenotype and insensitivity to the CYP51 inhibitor. WT T. b. brucei and $\mathrm{HpHbR}$ knock-out (KO) were incubated for $48 \mathrm{~h}$ with 0 to $5 \mu \mathrm{M}$ of ketoconazole. (g) T. b. rhodesiense Etat $1.2 \mathrm{R}$ cells freshly isolated from mice and transferred to in vitro culture conditions with either normal human serum containing $\mathrm{HpHb}(\mathrm{Hp}+/+)$, human serum lacking $\mathrm{Hp}$ (anhaptoglobinemic) (Hp-/-), or anhaptoglobinemic serum complemented with purified human $\mathrm{Hp}(\mathrm{Hp}-/-+\mathrm{Hp})$. Their growth rate was determined after incubation for $48 \mathrm{~h}$ with 0 to $5 \mu \mathrm{M}$ of ketoconazole. 
a

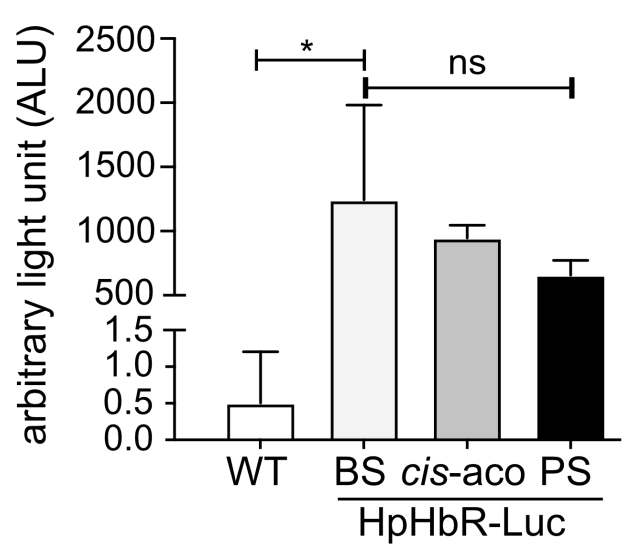

C

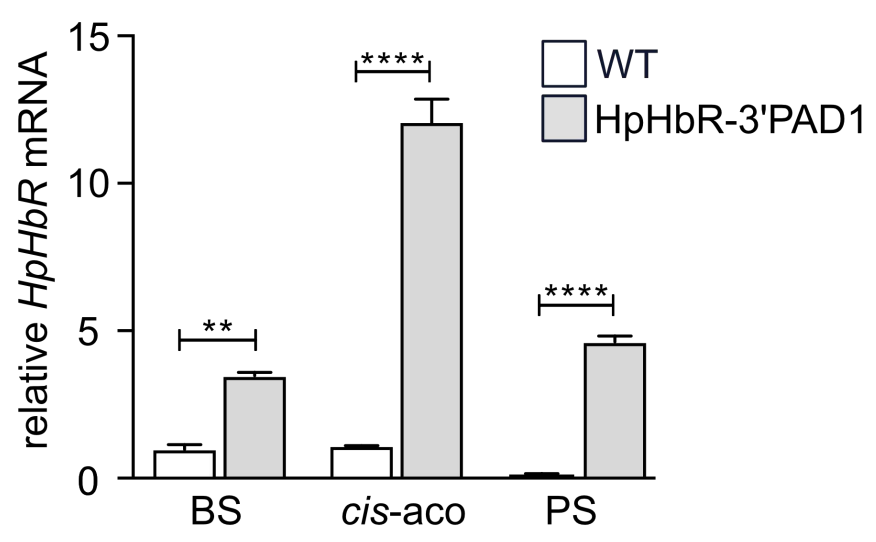

e

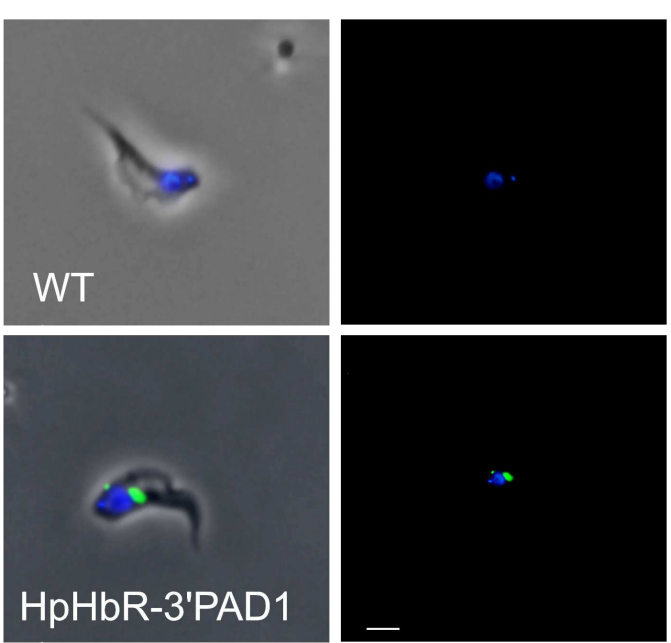

phase / DAPI / Hp-Alexa 488

g

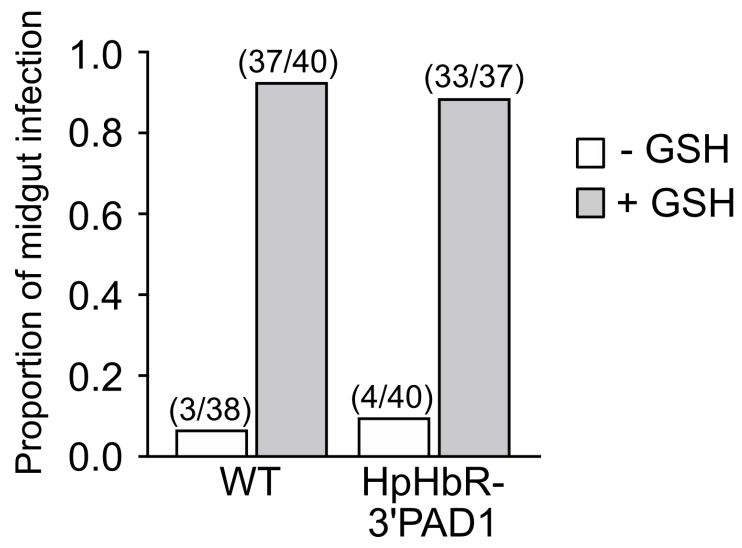

b

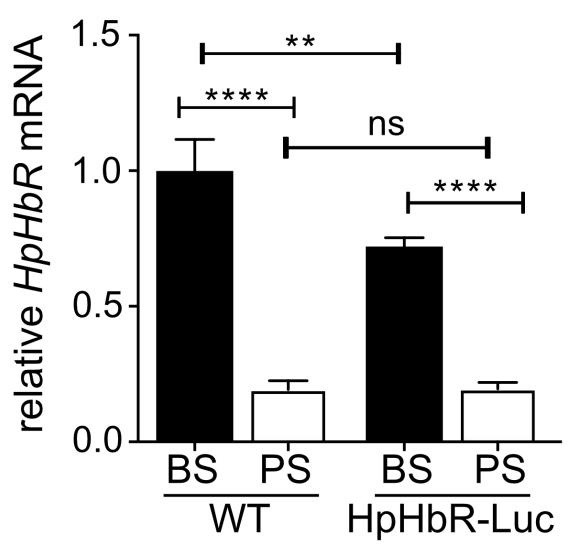

d

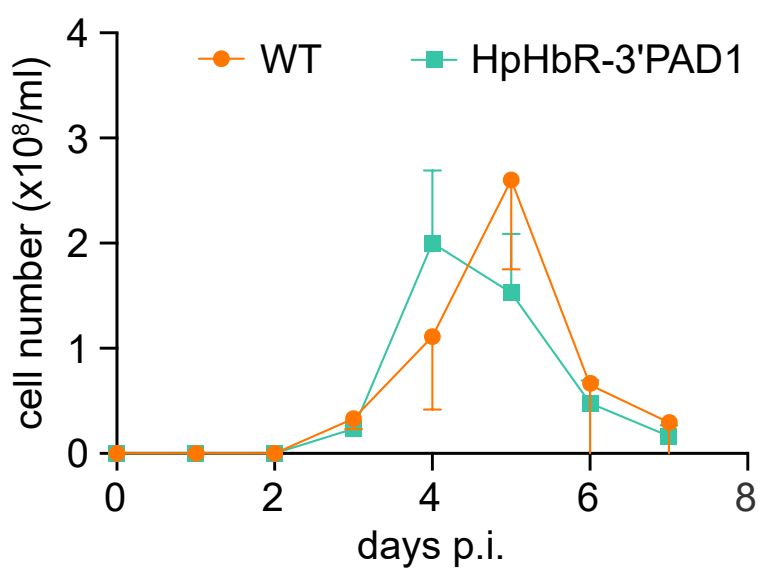

f

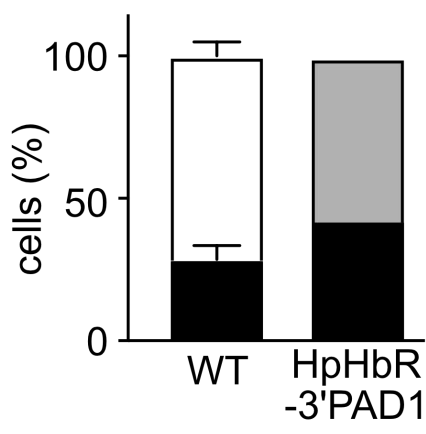

$\square$ SL

$\square$ ST Hp-Alexa 488-

$\square$ ST Hp-Alexa $488^{+}$ 
Figure 3. Artificial expression of $\mathrm{HpHbR}$ in stumpy form does not interfere with life cycle progression

(a) T. b. brucei engineered to express Luciferase along with $\mathrm{HpHbR}$ at the end of the polycistronic transcription unit ( $\mathrm{HpHbR}$-Luc). Luciferase activity was measured during in vitro differentiation in the bloodstream stage (BS), $2 \mathrm{~h}$ after differentiation was triggered by cisaconitate (cis-aco), and the procyclic stage (PS), as well as in wild types (WT) used as a control. (b) RT-PCR assessed the expression of $\mathrm{HpHbR}$ in WT and HpHbR-Luc cells. Complementary DNA was synthesized from RNA extracted from the bloodstream (BS) and procyclic stages (PS) and used for RT-PCR as described in experimental procedures. (c) The expression of $\mathrm{HpHbR}$ was assessed as in (b) in the WT T. b. brucei and HpHbR-3'PAD1 trypanosomes. RNA was extracted from the BS, $2 \mathrm{~h}$ after differentiation was triggered by cisaconitate (cis-aco) and the procyclic stage (PS). (d) In vivo infectivity of WT T. b. brucei and $\mathrm{HpHbR-3'PAD1}$ cells was evaluated by infecting mice with $1 \times 10^{4}$ cells. The parasitemia was counted daily till day 7. (e) A representative WT T. b. brucei and HpHbR-3'PAD1 cell isolated from the blood of mice; the latter ones internalized the fluorescently-labeled $\mathrm{HpHb}$ complex (green). (f) Based on morphology and the $\mathrm{HpHb}$ complex uptake, the same cells as in (e) were categorized as slender (SL) or stumpy (ST), the latter with the $\mathrm{HpHb}$ complex internalized (Hp-Alexa $488^{+}$) or not (Hp-Alexa 488). All experiments above $(n=3)$ were analyzed for significant differences using Student's $t$-test (ns, $p>0.05 ;{ }^{*}, p \leq 0.05 ;{ }^{* *}, p \leq 0.01$; $\left.{ }^{* * * *}, p \leq 0.0001\right)$. (g) The capacity to establish midgut infection in tsetse flies was determined for WT T. b. brucei and HpHbR-3'PAD1 cells isolated from the blood of mice. Bloodstream stage parasites were administrated in the fly's first bloodmeal without (-GSH) or with supplementation of $10 \mathrm{mM}$ glutathione (+GSH). 
a

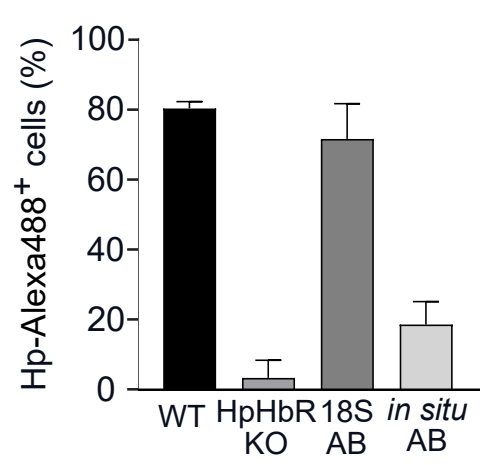

b

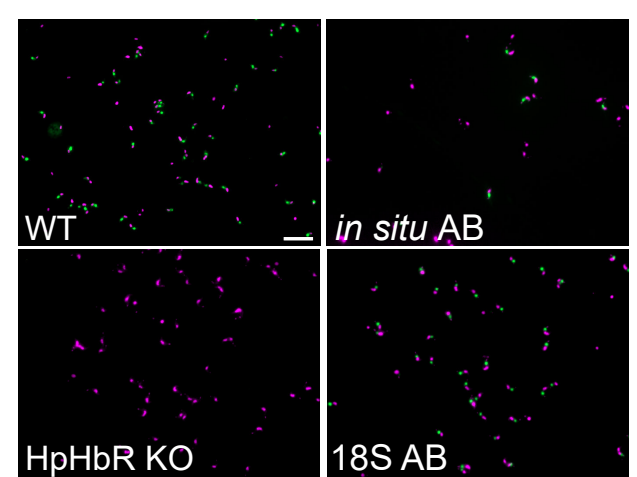

phase / DAPI / Hp-Alexa 488

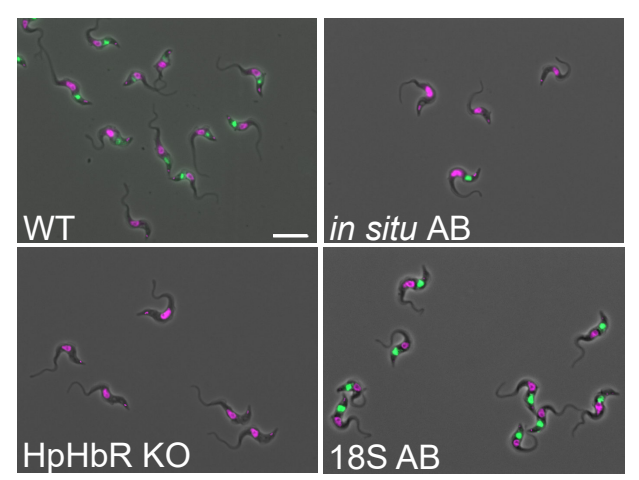

phase / DAPI / Hp-Alexa 488
C

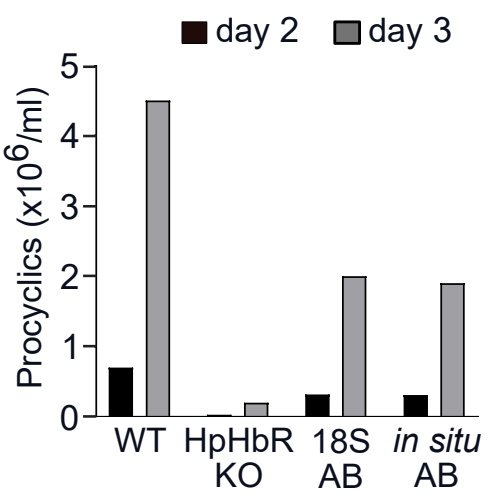

d

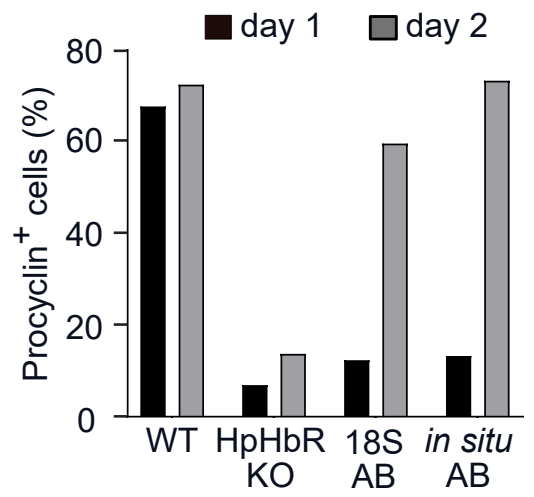

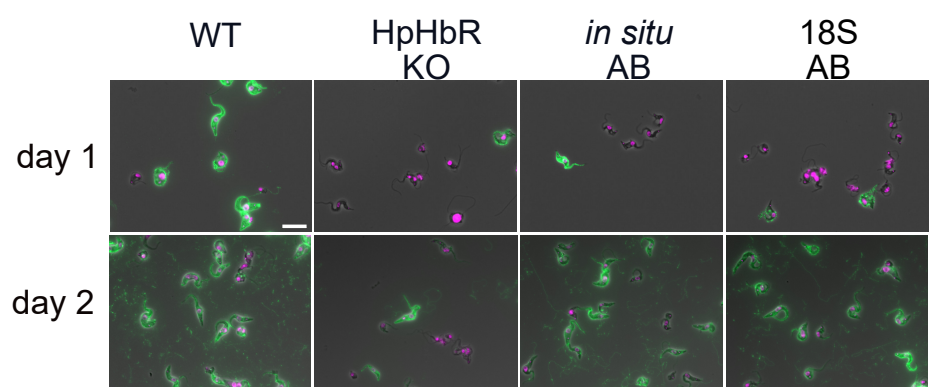

phase / DAPI / Procyclin e

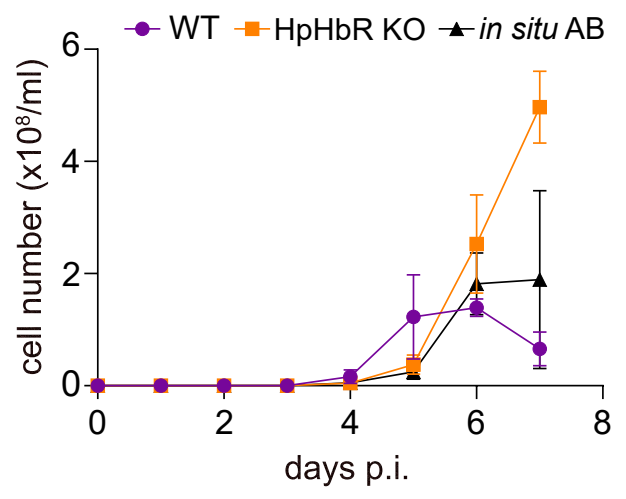

g

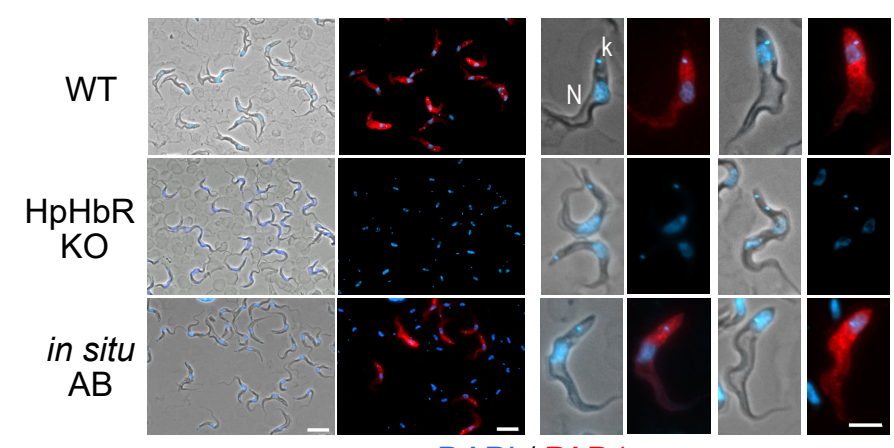

DAPI / PAD1 f
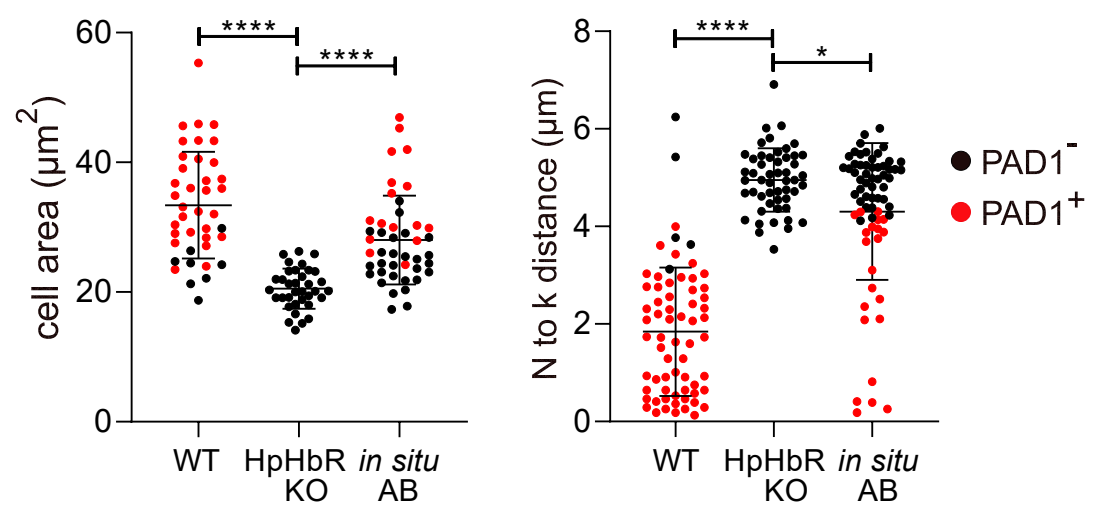

h

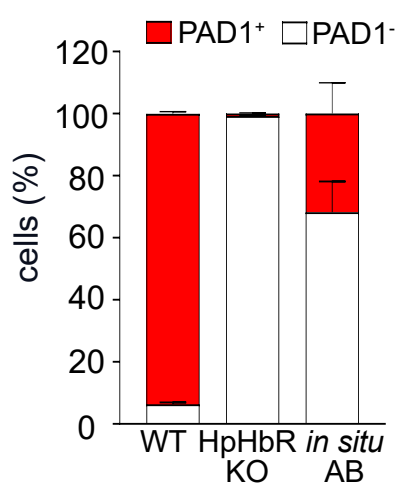

i

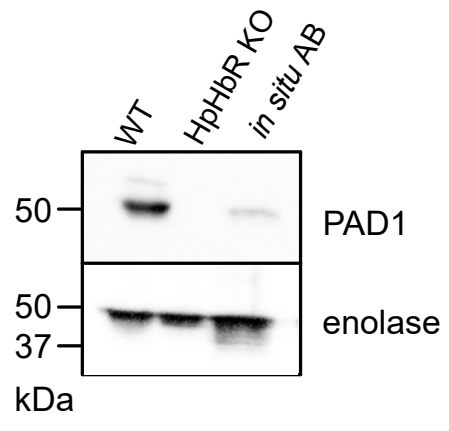




\section{Figure 4. Slender-to-stumpy form differentiation is disrupted in HpHbR knock-out}

cells

(a) The uptake of fluorescently-labeled haptoglobin $(\mathrm{Hp})$ was monitored in WT T. b. brucei, $\mathrm{HpHbR}$ knock-outs ( $\mathrm{HpHbR} \mathrm{KO}$ ), as well as in add-back cells, in which $\mathrm{HpHbR}$ was

expressed either from the 18S rRNA locus (18S AB), or the in situ locus (in situ AB). All cells were harvested 4 days post-infection and mixed and incubated for $2 \mathrm{~h}$ with Hp-A488; $(n=2)$

(b) Fluorescence microscopy of cell lines as in (a), with labeled Hp (green) and DNA visualized by DAPI (magenta). Note the absence of $\mathrm{Hp}$ uptake in $\mathrm{HpHbR}$ knock-outs. Left panel: Low magnification of the purified cells (scale bar, $20 \mu \mathrm{M}$ ); Right panel: Cells at higher magnification (scale bar, $10 \mu \mathrm{M}$ ). (c) In vitro differentiation of cell lines as in (a). Cells were placed to DTM medium with $3 \mathrm{~mm}$ sodium citrate/cis-aconitate at $27^{\circ} \mathrm{C}$ and procyclic cells were counted on day 2 and 3; $(n=3)(d)$ Examination for procyclin expression on day1 and day 2 Left panel: Percentage of procyclin positive cells (procyclin ${ }^{+}$) established by fluorescence microscopy with procyclin antibody (green) and DAPI (magenta). Right panel: Representative pictures for each cell line; $(n=3)(\mathbf{e})$ In vivo infectivity of WT T. b. brucei, $\mathrm{HpHbR} \mathrm{KO}$, and the in situ $\mathrm{AB}$ was evaluated by infecting mice with $1 \times 10^{4}$ cells. The parasitemia was counted daily till day 7 when the experiment was terminated. Parasites from infections were harvested on day 7 post-infection and separated from blood using the DEAE column. (f) Morphological characterization of cell lines described in (e), containing the PAD1positive ST forms (red dots) along with the PAD1-negative (SL) cells (black dots). Left panel: cell area; Right panel: the distance between the nucleus $(\mathrm{N})$ and the kinetoplast $(\mathrm{k})$. Results were analyzed for significant differences using Student's $t$-test $\left({ }^{*}, p \leq 0.05 ;{ }^{* * *}, p \leq 0.0001\right)$. (g) Indirect immunofluorescence with PAD1 antibody (red), which is specific for ST Left panel: Low magnification of the purified cells (scale bar, $10 \mu \mathrm{M}$ ); Right panel: Individual cells at high magnification (scale bar, $5 \mu \mathrm{M}$ ), with discernible DAPI-stained nucleus $(\mathrm{N})$ and kinetoplast (k). (h) Quantification of PAD1-positive and PAD1-negative cells (ex vivo, day 7 p.i.) described in (e). In the columns, the stumpy form is shown in red. (i) Western blot analysis with PAD1 antibody of cell lines described in (e). Enolase antibody was used as a loading control. 
a

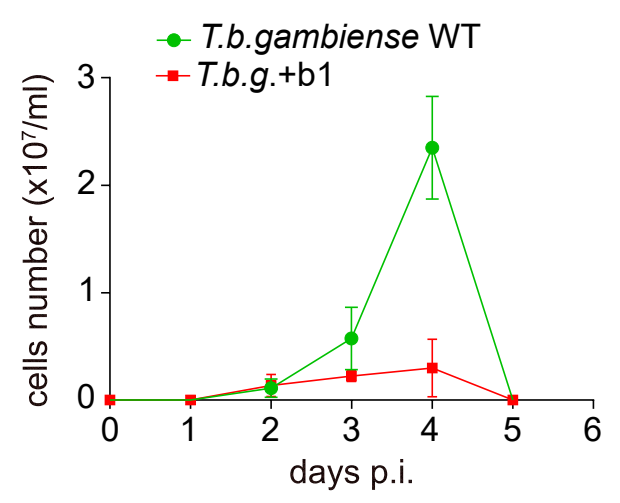

b

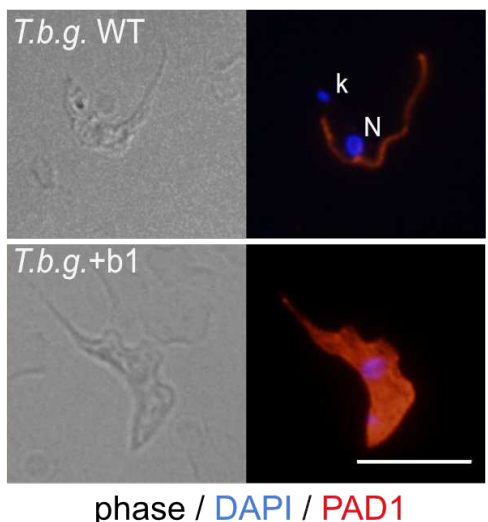

C

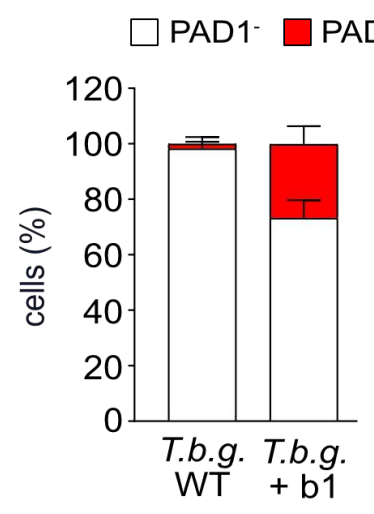

d

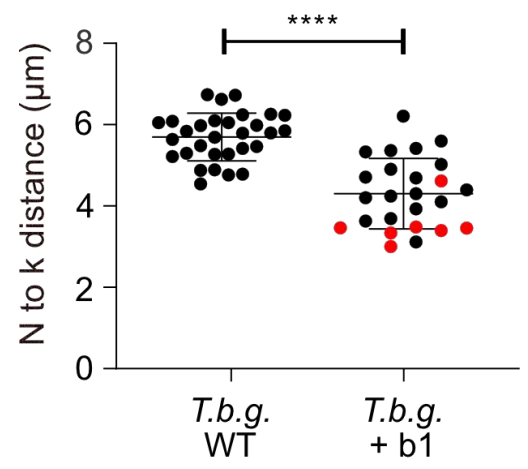

$\mathbf{f}$

T.b.g. FIELD d4 p.i.

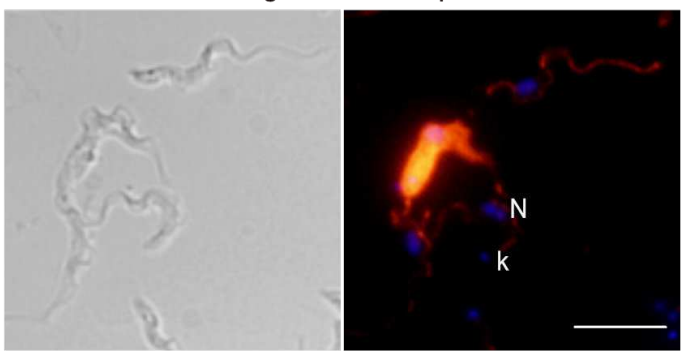

phase / DAPI / PAD1

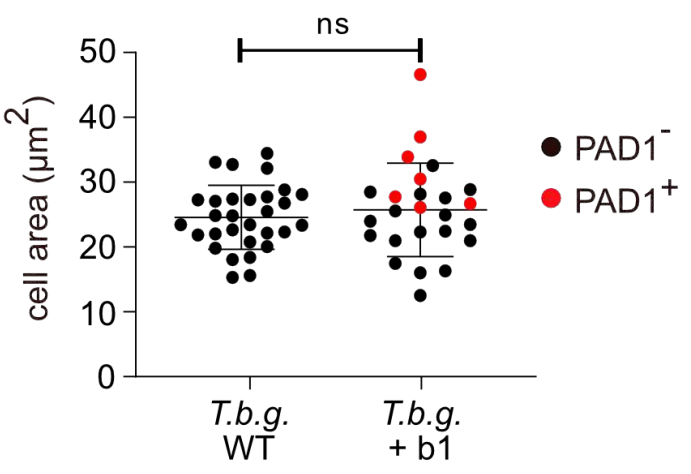

g

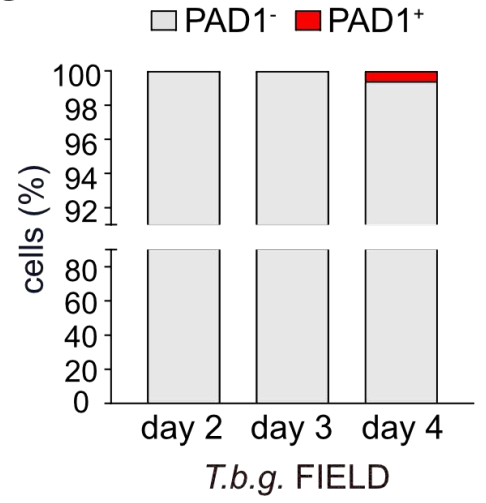

e

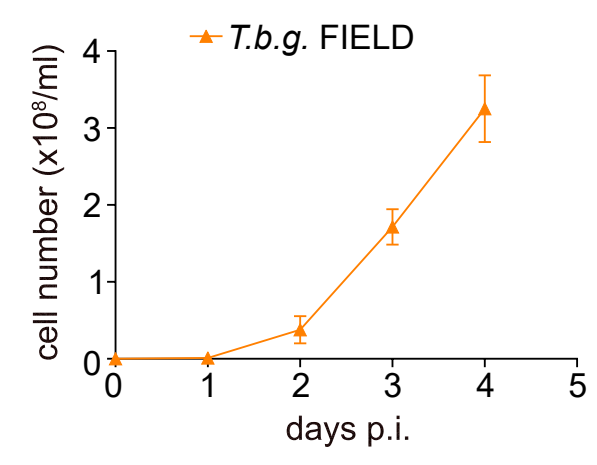

h

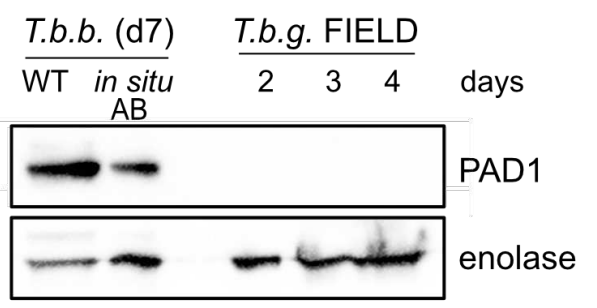

\section{Figure 5. Restoration of stumpy formation in T. b. gambiense}

(a) In vivo infectivity of WT T. b. gambiense LiTat 1.3 (green line) and T. b. gambiense expressing T. b. brucei HpHbR from the $18 \mathrm{~S}$ rRNA locus (T. b. g. + b1) (red line) was evaluated by infecting mice with $3 \times 10^{6}$ cells; $n=3$.

The parasitemia'was counted daily till day 6, when the experiment was terminated. (b) Indirect immunofluorescence with PAD1 antibody (red), which is specific for ST. DNA in the nucleus (N) and kinetoplast (k) was stained with DAPI (blue). Scale bar, $5 \mu \mathrm{m}$. (c) Quantification of PAD1-positive and PAD1-negative cells (ex vivo, day 4 p.i.) described in $(a)$; $(n=3)$. In the columns, the stumpy form is shown in red. (d) Morphological characterization of cell lines described in (a), containing the PAD1-negative cells (black dots) or PAD1-positive cells (red dots). Left panel: the distance between the nucleus $(\mathrm{N})$ and the kinetoplast $(\mathrm{k})$; Right panel: cell area. Results were analyzed for significant differences using Student's $t$-test (ns, p0.05; $\left.{ }^{* * * *}, \mathrm{p} \leq 0.0001\right)$. (e) In vivo infectivity of $T$. b. gambiense Bosendja Field strain. The parasitemia was counted daily till day 4, when the experiment was terminated.; $(n=3)(f)$ Immunofluorescence using PAD1 antibody, which reveals a fraction of the PAD1-positive cells. DNA in the nucleus $(N)$ and kinetoplast (k) was stained with DAPI (blue). Scale bar, $5 \mu \mathrm{m}$. (g) Quantification of PAD1-positive and PAD1-negative cells in the T. b. gambiense Field strain ex vivo days 2-4 p.i. (h) PAD1 antibody Western blot analysis of WT T. b. brucei and in situ AB, as well as T. b. gambiense Field strain. Enolase antibody was used as a loading control. 
Figures

a
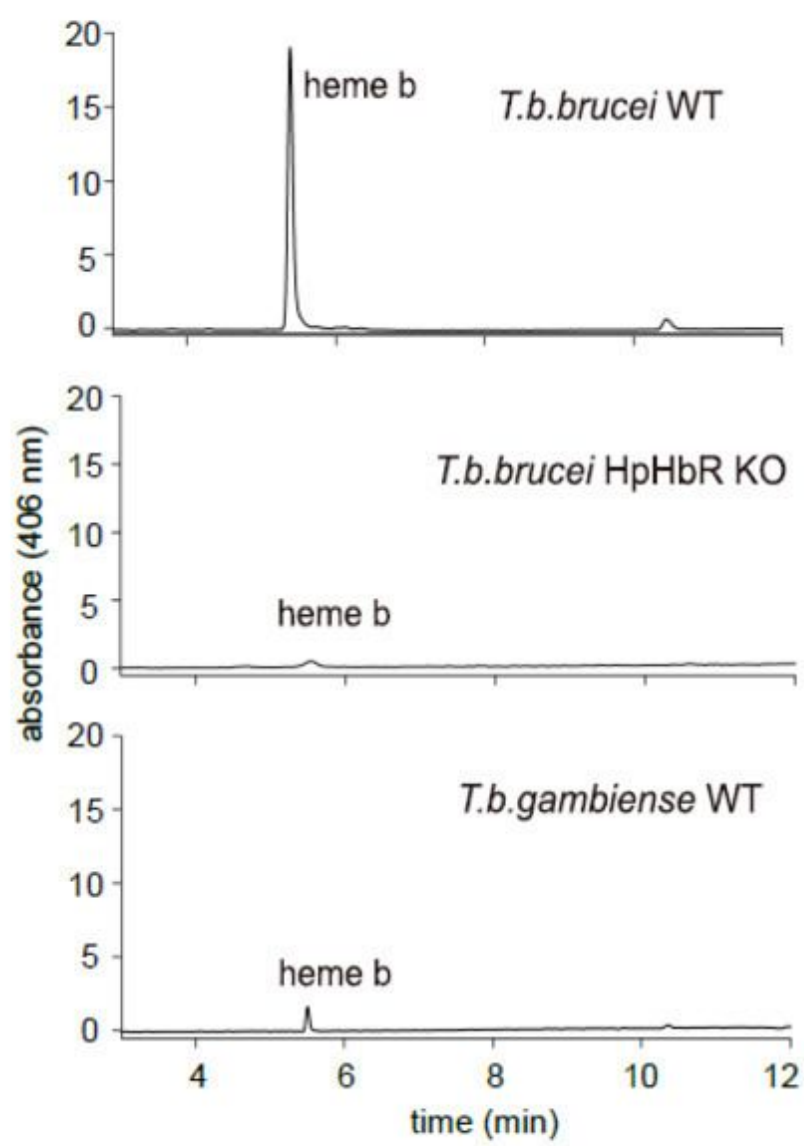

f

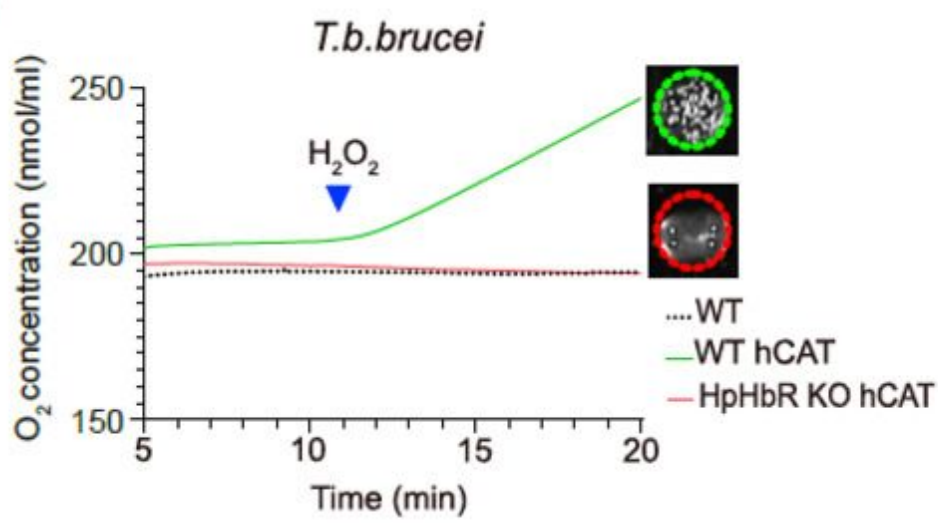

b
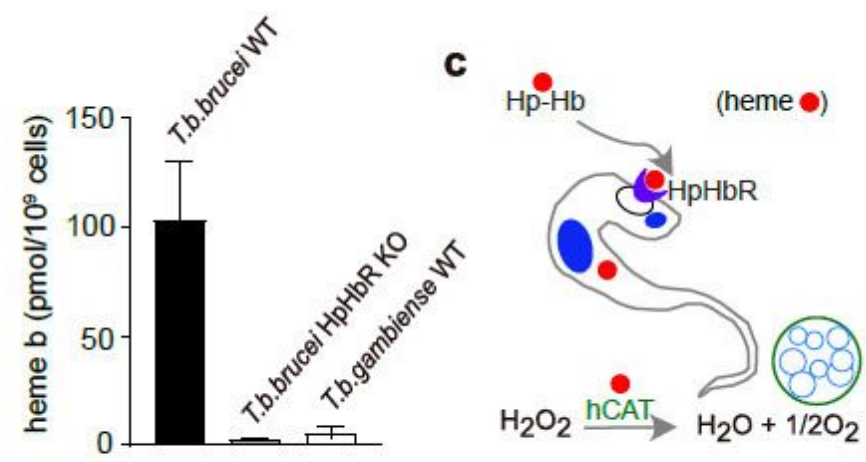

d

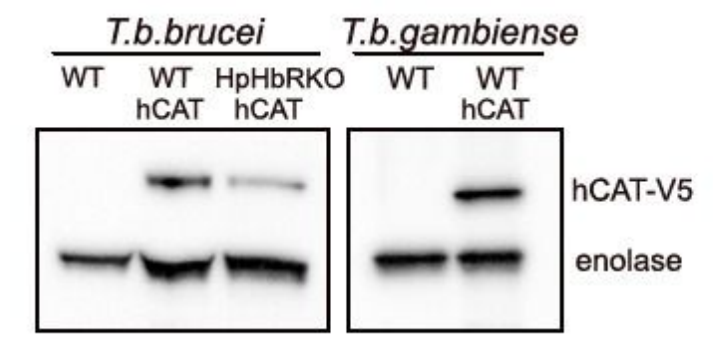

e

T.b.brucei
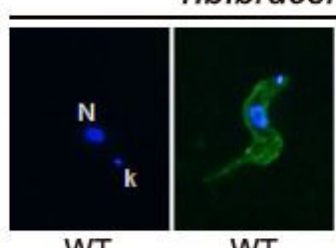

WT
hCAT

hCAT ' hCAT T.b.gambiense

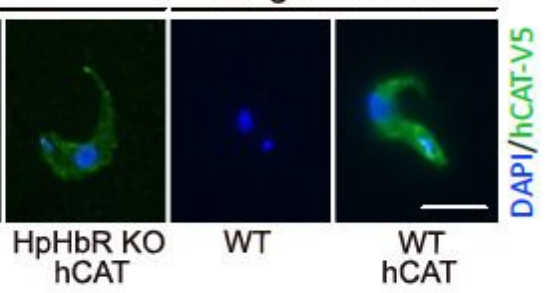

hCAT
T.b.gambiense

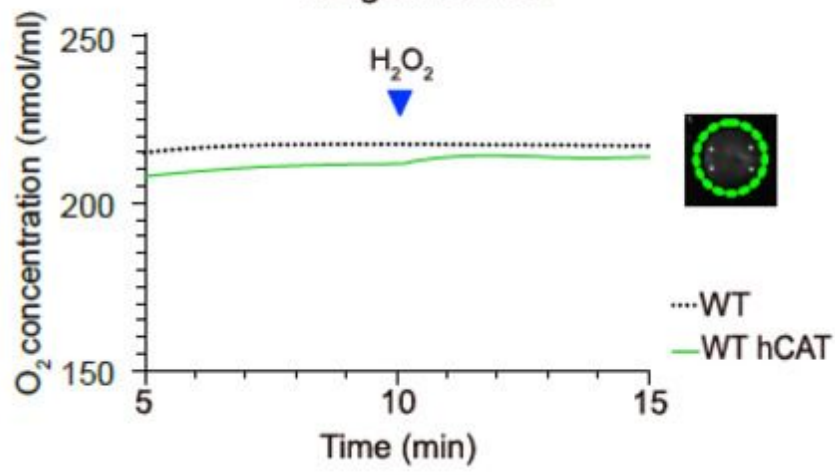

Figure 1

Detection of heme and hemoproteins in bloodstream stages of T. b.brucei and T. b. gambiense 
a
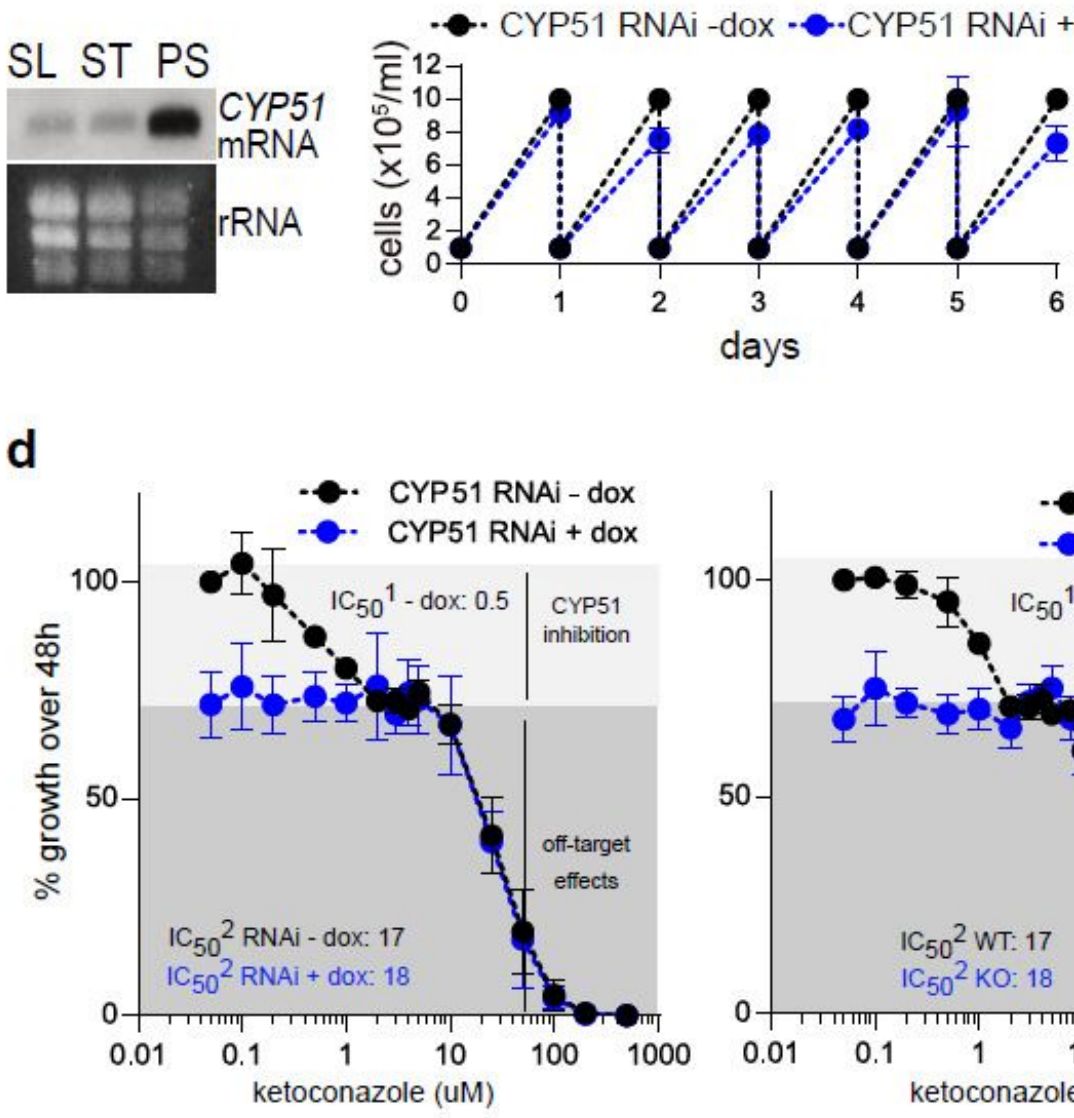

days
C

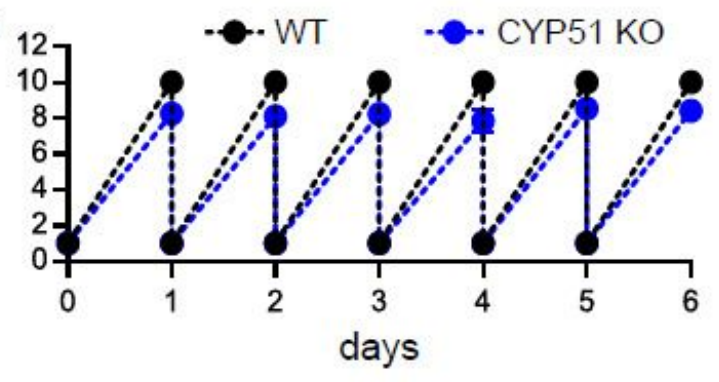

e
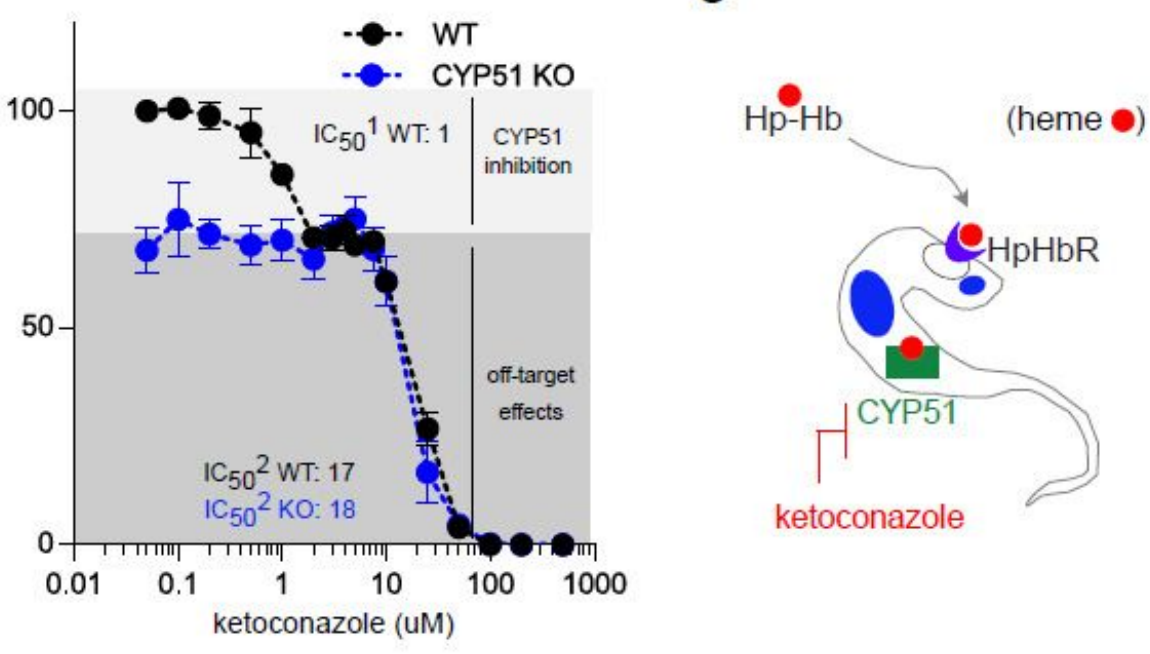

ketoconazole f

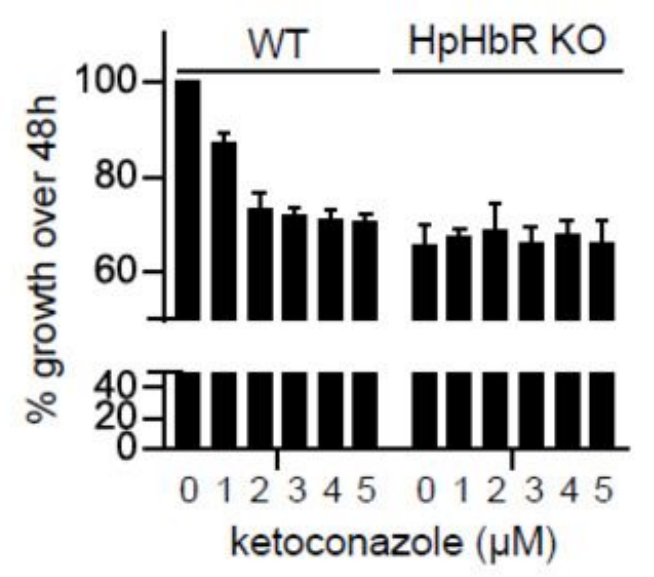

g

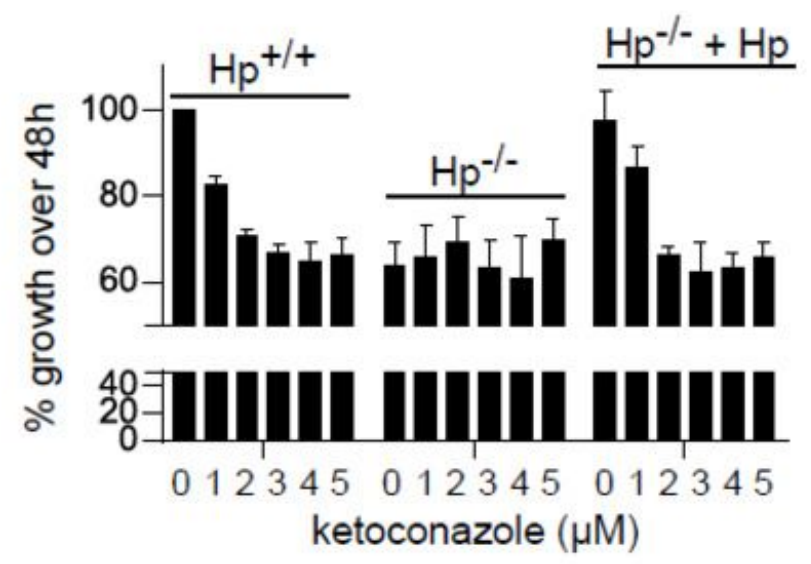

Figure 2

Effect on growth and ketoconazole sensitivity after CYP51 invalidation in the bloodstream stage 
a

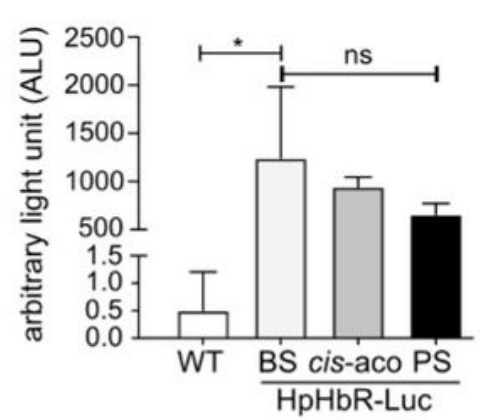

C

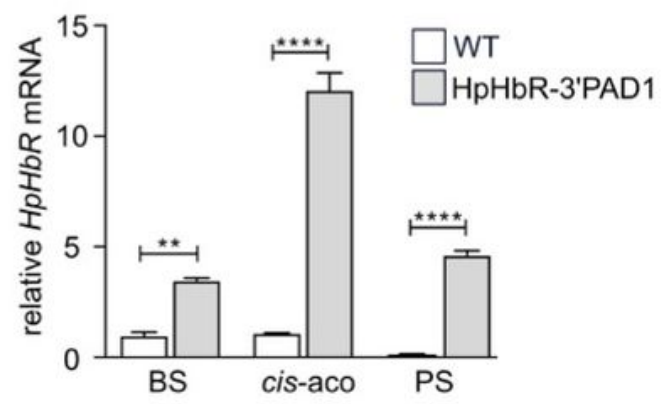

e
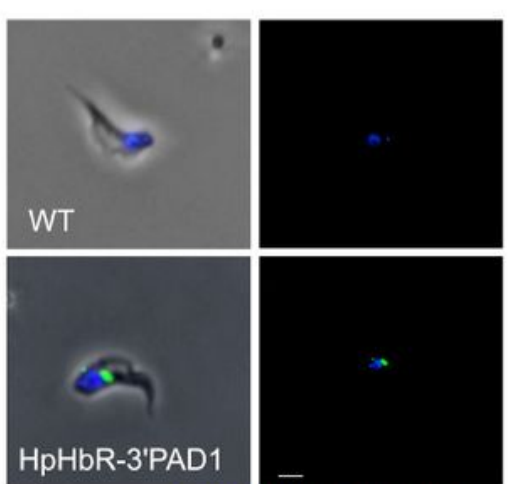

phase / DAPI / Hp-Alexa 488 b

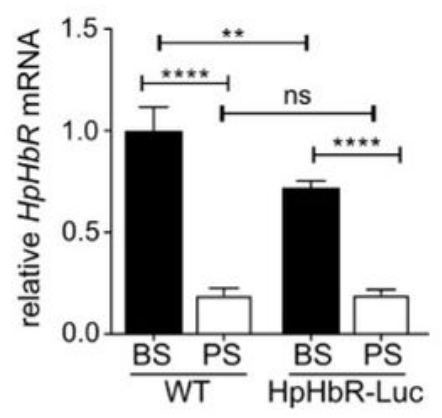

d

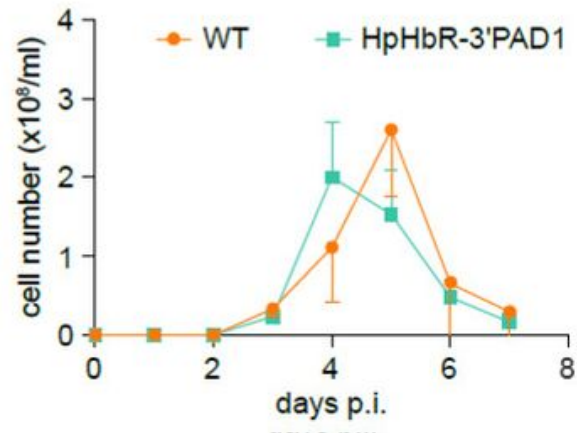

f

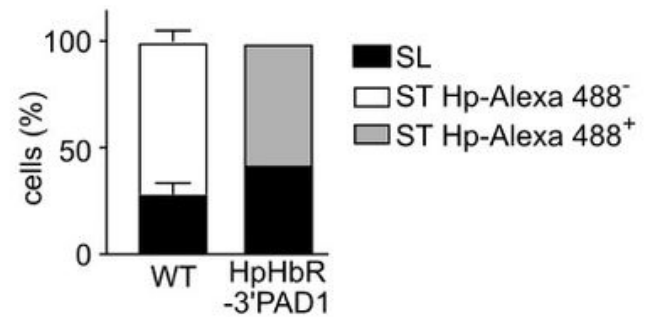

g

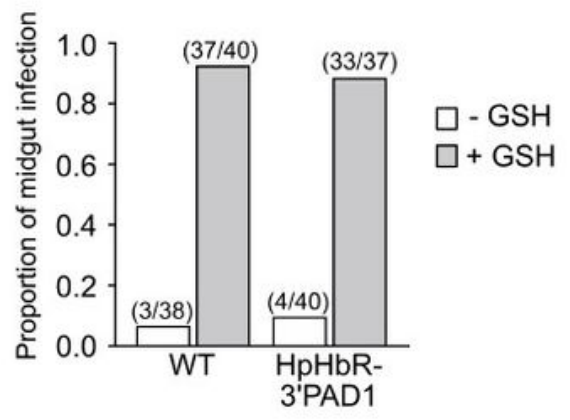

Figure 3

Artificial expression of $\mathrm{HpHbR}$ in stumpy form does not interfere with life cycle progression 
a

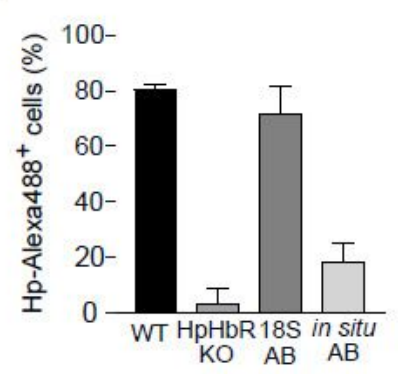

b

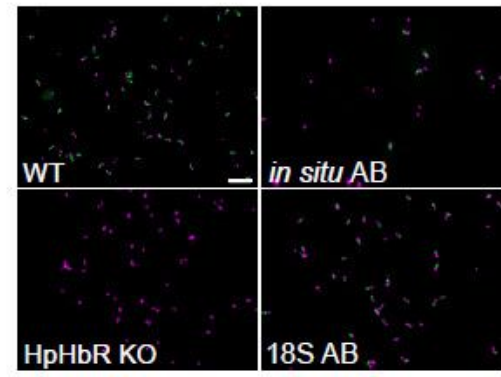

phase / DAPI / Hp-Alexa 488

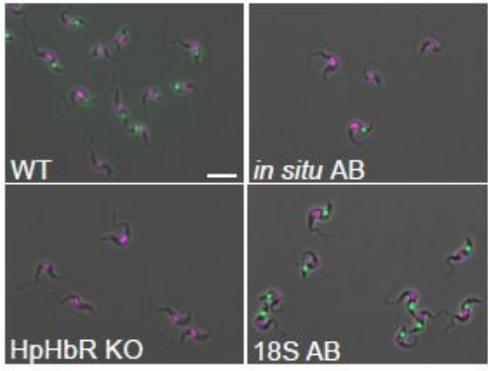

phase / DAPI / Hp-Alexa 488
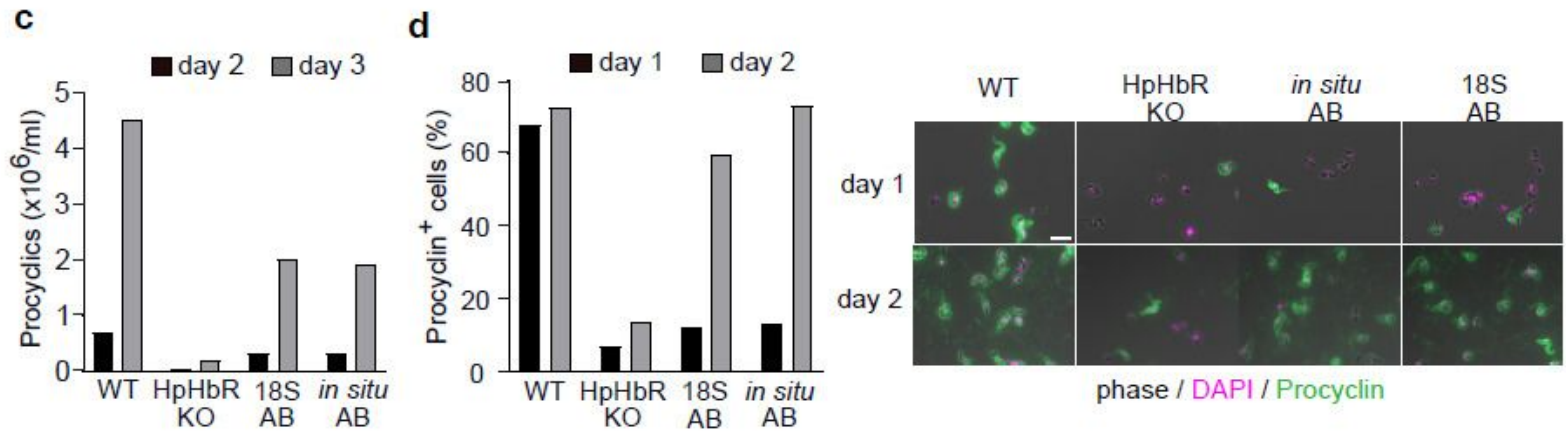

phase / DAPI / Procyclin

e

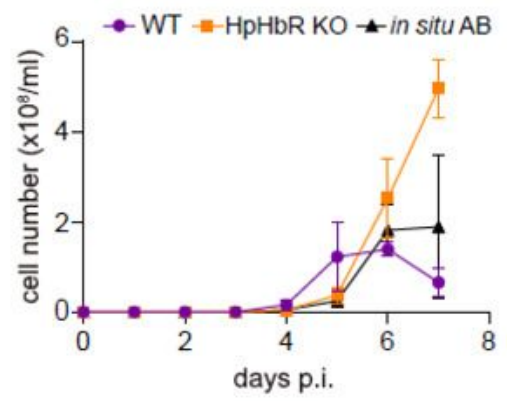

g

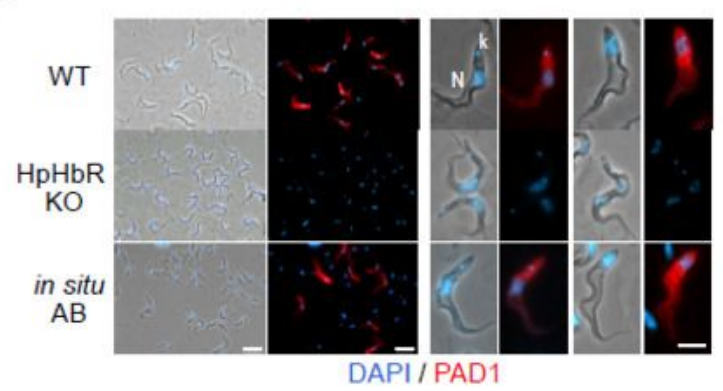

f
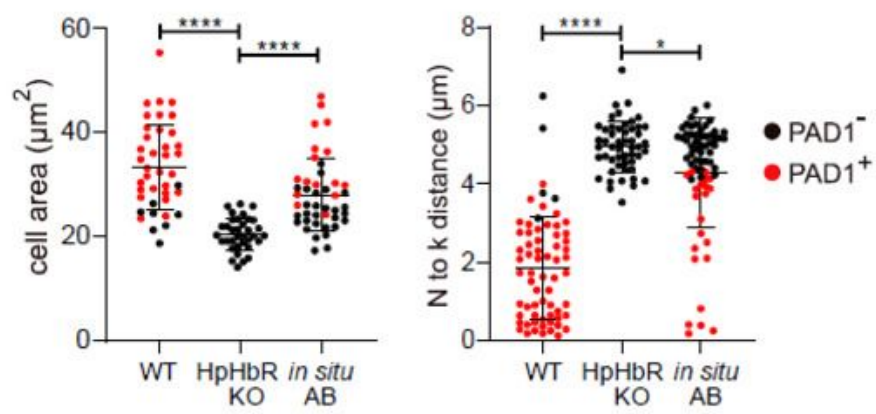

h

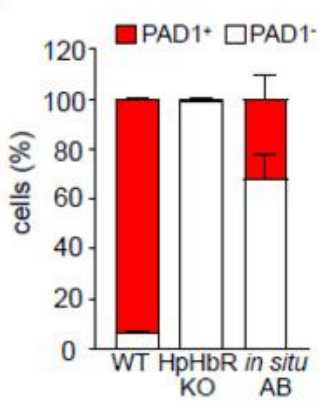

i

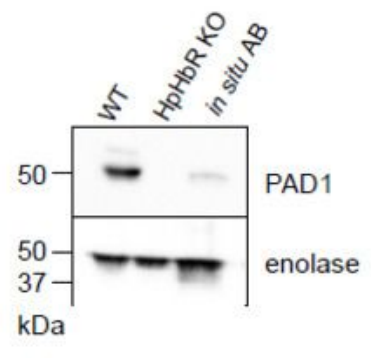

\section{Figure 4}

Slender-to-stumpy form differentiation is disrupted in $\mathrm{HpHbR}$ knock-out cells 
a

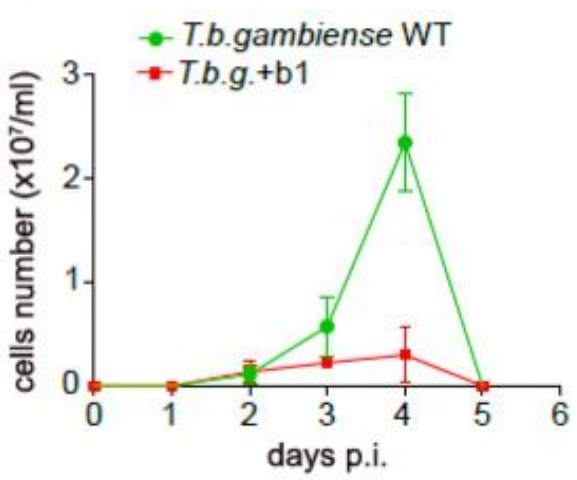

d

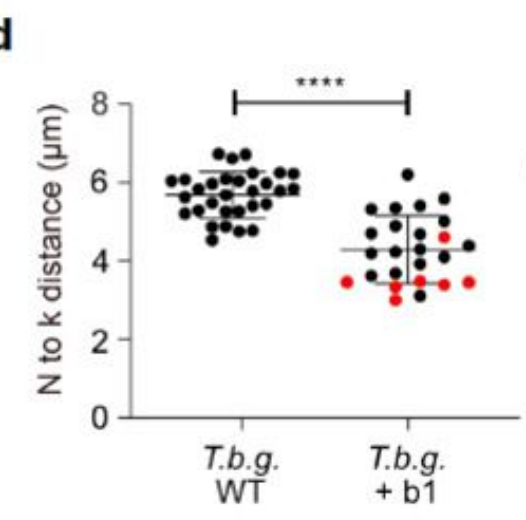

$\mathbf{f}$

T.b.g. FIELD d4 p.i,

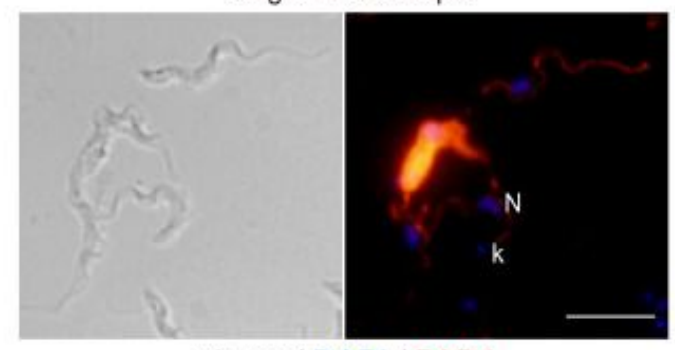

phase / DAPI / PAD1 b

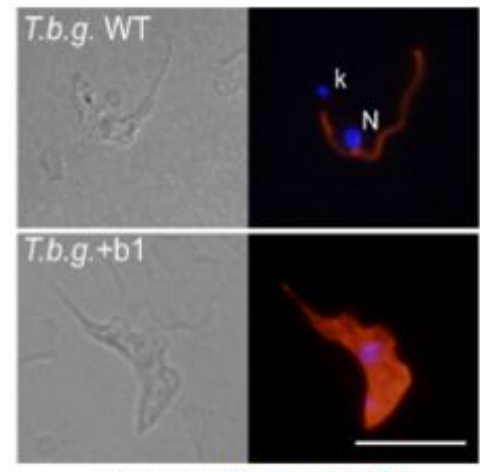

phase / DAPI / PAD1
C

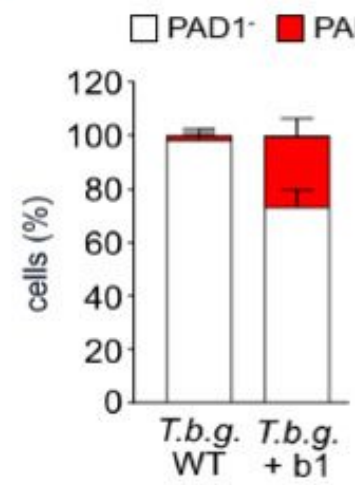

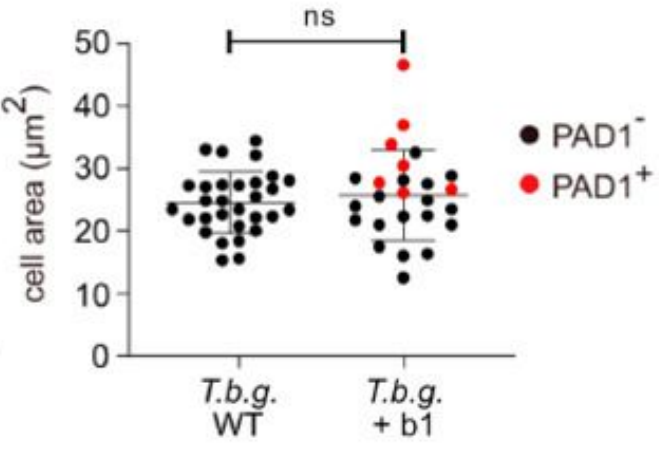

g

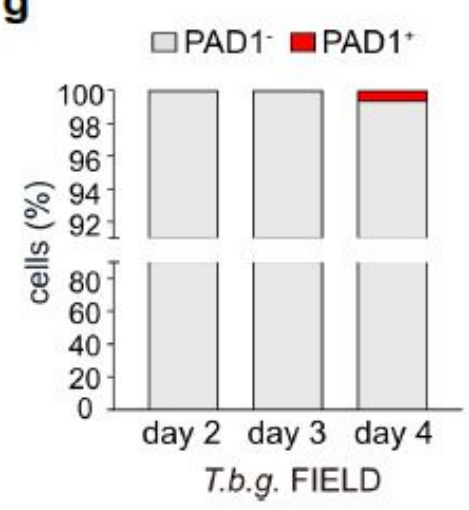

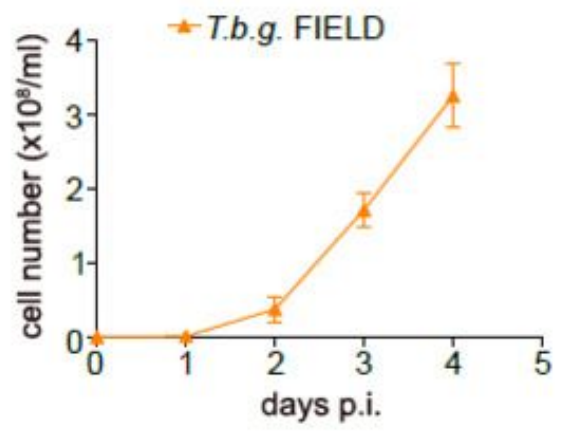

h

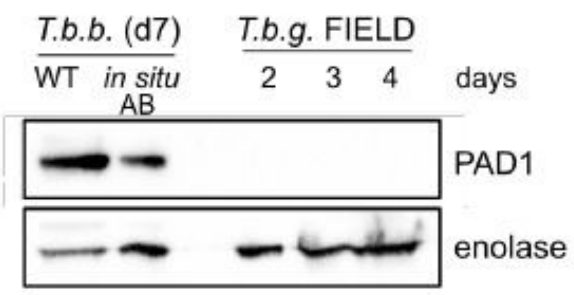

\section{Figure 5}

Restoration of stumpy formation in T. b.gambiense (a) In vivo infectivity ofWT T. b.gambiense LiTat1.3(greenline)and

\section{Supplementary Files}

This is a list of supplementary files associated with this preprint. Click to download.

- HorakovaHpHbRSupplementary.pdf 